\title{
Theologische Diskussion und Entscheidung auf Synoden: Verfahrensformen und -erwartungen
}

\author{
[PRE-EDIT]
}

Die Synoden im trinitarischen Streit, Ed. by Heil, Uta / Stockhausen: Texte und Untersuchungen zur Geschichte der altchristlichen Literatur 177

Wie wird auf Synoden Theologie diskutiert und Rechtgläubigkeit definiert? Was macht Synoden zum geeigneten Instrument oder Forum dieses Bemühens? Oder sollte man synodale Zusammenkünften nicht prinzipiell als Ereignisse begreifen, die durch politische Machenschaften, Machtkämpfe und persönliche Rivalitäten geprägt und darum ganz ungeeignet sind, in angemessener Weise hochkomplexe und fundamental wichtige Fragen christlicher Theologie zu bearbeiten, geschweige denn bindend zu beantworten. ${ }^{1}$ Verschärft werden solche möglichen Bedenken angesichts eines zweifellos erheblich variierenden Maßes an intellektueller Begabung und formaler philosophischer, rhetorischer und dialektischer Bildung zwischen den Beteiligten. Nicht erst moderne Forscher äußern den Verdacht oder Vorwurf, daß es sich bei den Versammelten zum guten Teil um ungebildete Leute handelte, ebenso unvorbereitet wie unwillig sich in ernsthafter Diskussion auseinanderzusetzten, und die darum eben doch aufs ganze gesehen den jeweils dominierenden Gestalten oder dem politischen Willen der Herrscher schlicht folgten. Eine kritische Analyse von synodalen Entscheidungen wird damit in zwei übergeordneten Fragerichtungen nötig. Zum einen gilt es, die intellektuelle Valenz und theologische Tragfähigkeit einzelner synodaler Texte zu untersuchen, und sie in den Kontext der theologischen Diskussionen ihrer Zeit einzuzeichnen. Diesen Fragen haben sich zahlreiche theologiegeschichtliche Arbeiten mit reichem Ertrag gewidmet. Zum anderen gilt es aber auch, nach den Formen und Modi zu fragen, in denen Theologie auf Synoden zur Sprache kommt,

\footnotetext{
${ }^{1}$ Rein, oder doch wenigstens im Schwerpunkt, machtpolitisch motivierte und interessierte Interpretationen des Synodalgeschehens sind klassisch von Eduard Schwartz vorgelegt worden (s. von zahlreichen Einzelstudien zum Themenbereich exemplarisch die Beiträge E. Schwartz, Über die Reichskonzilien von Theodosius bis Justinian, in: Gesammelte Schriften, Bd. 4: Zur Geschichte der Alten Kirche und ihres Rechts, Berlin 1960, 111-58; ders.: Die Konzilien des IV. und V. Jahrhunderts, HZ 104 (1910), 1-37; ders.: Zur Vorgeschichte des ephesinischen Konzils, HZ 112 (1914) 237-63) und werden in je verschieden nuancierter Form als kritische Perspektive an die Geschehnisse nach wie vor herangetragen. Von einer solchen Warte aus stellt sich die Frage nach der Eignung von Synoden zur Entscheidung über doktrinäre Inhalte natürlich nicht - oder ist bereits negativ entschieden -, wird doch die theologische Thematik der Auseinandersetzung lediglich als Vehikel zum Austrag für dahinter verborgene, gesellschaftliche und persönliche Konflikte und Ambitionen begriffen.
} 
und zu untersuchen, welche zusätzlichen Faktoren gegebenenfalls im synodalen Geschehen bei dem Versuch wirksam werden, verbindliche Entscheidungen herbeizuführen. Diesen Fragen hat sich die Forschung bei aller Aufmerksamkeit auf die jeweiligen historischen Umstände im Einzelnen bislang nicht in gleicher Intensität gewidmet. Beide Fragerichtungen, nach den expliziten oder impliziten Regeln theologischer Diskussion auf Synoden und nach den Normen und Kriterien der inhaltlichen Bestimmung von Orthodoxie, sind aufs engste miteinander verwoben, aber nicht einfach identisch. Woran sich Orthodoxie in der Sache ausweist, ist kaum einfach auf die Frage nach einem - anhand welchen Maßstabs auch immer „korrekt“ zu nennenden Ablauf der Diskussion zu verschieben. Die Frage nach den theologischen Begründungszusammenhängen und Argumentationsweisen verbindlicher Lehrbildung in den trinitarischen und christologischen Streitigkeiten bleibt an die theologiehistorische Forschung verwiesen. Im Folgenden soll es einzig um die Verfahrensdimension des synodalen Geschehens gehen, d.h. um die Fragen nach dem Ort und der Gestalt theologischer Diskussion und nach dem Wirksamwerden von anderen als intellektuell-diskursiven Verhaltensweisen, Strategien und Kommunikationsmöglichkeiten im synodalen Zusammenhang, sei es in Ergänzung oder sei es in Konkurrenz zur theologischen Debatte. Dabei werden exemplarisch zwei Synoden, situiert etwa in der Mitte des vierten Jahrhunderts, also auf einem Höhepunkt synodaler Aktivität, ins Auge gefaßt und auf ihre Verfahrensformen hin analysiert.

Für eine solche Untersuchung bedarf es der kritischen Reflexion auf die Parameter der Analyse von synodalen Verfahrensweisen und auf die Maßstäbe ihrer Beschreibung und Beurteilung. Vor kurzem hat ein Forscher das synodale Geschehen als „Voting about God“ - „Abstimmen über Gott“ dargestellt ${ }^{2}$. Dies suggeriert eine Perspektive quasi-demokratischer Mehrheitsfindung, aus der sich die Synoden der Alten Kirche dann als in vielfältiger Hinsicht mangelhaft herausstellen. Machtausübung, Aberglaube und intellektuelle Inkompetenz gepaart mit einer unverhältnismäßigen Aufmerksamkeit auf und Beanspruchung von obskuren

\footnotetext{
${ }^{2}$ Ramsay MacMullen, Voting about God in Early Church Councils, New Haven / London (Yale University Press) 2006. Vgl. dazu etwa meine Rezension in: Zeitschrift für Antikes Christentum 12 (2008) 172-174 und L. Ayres, Majority Rules, in: First Things, December 2007, [http://www.firstthings.com/article/2007/11/001-majority-rules; last accessed 3.8.2012].
} 
theologischen Quisquilien konspirieren danach gegen eine wirkliche, sachlich wie formal angemessene Beteiligung aller an den Entscheidungsprozessen.

Will man nicht in dieser Weise anachronistische Maßstäbe von demokratischer Partizipation oder hierarchieloser Diskursivität bei der Analyse synodaler Abläufe und ihrer Leistungsfähigkeit ansetzen ${ }^{3}$, so ist vielmehr zu fragen, was Zeitgenossen von Synoden erwarteten, und aufgrund welcher Normen und Standards sich ihnen die Überzeugung vermittelte, daß bei einer bestimmten Gelegenheit Theologie in methodisch solider und der Sache angemessener Weise betrieben worden sei oder, formaler beschrieben, daß Verbindlichkeit in einer Weise erreicht wurde, die alle Beteiligten mitzutragen in der Lage waren. Es geht also um die Fragen nach Regelhaftigkeit als einer möglichen Anforderung an die Valenz synodaler Abläufe im Grundsatz einerseits, und spezifisch nach der Spanne der Interaktionsformen und Kommunikationsmöglichkeiten, mit deren Hilfe eine Problematik bearbeitet und ein Konflikt zum Austrag und zur Lösung kommen konnte, andererseits. Für die erste Frage bedürfte es einer Analyse der „Konzilsidee“ als einer philosophischtheologischen Konstruktion der Autoritätsmerkmale von Synoden im Rahmen der Ekklesiologie und ggf. Pneumatologie sowie der Untersuchung, inwieweit diese Theoreme eine Reflexionen auf Regelhaftigkeit einschließen bzw. verlangen. ${ }^{4}$ Für die zweite, hier angezielte, sollen nachfolgend eine Reihe von Dokumenten des vierten Jahrhunderts in ein kritisches Gespräch mit einer modernen Verfahrenstheorie gebracht werden.

\footnotetext{
${ }^{3}$ Ersteres geschieht ohne eingehende ausdrückliche Reflexion bei MacMullen (s. vorigen Anm.), wenn Vorstellungen von Abstimmung und Mehrheit benutzt werden und nach dem Beitrag des 'ordinary bishop' in dieser Perspektive gesucht wird (s., apodiktisch, p. 2: “... how was the Christian consensus arrived at? The answer, well known, is: by majority vote of group leaders in occasional assemblies". Für differenziertere Vorstellungen der praktischen Umsetzung des 'democratic element' (12) vgl. jedoch 12-23.) Indirekt stehen Idealvorstellungen hierarchieloser Diskussion (und entsprechende Idealisierungen platonischer Dialoge, die sich bei näherer Betrachtung keineswegs hierarchielos präsentieren) hinter der weiter gehenden Kritik an einer angeblich fehlenden Dialogbereitschaft der Christen in der Antike schlechthin - ohne daß dabei Synoden speziell in den Blick gerieten (so etwa die Grundthese des im Sammelband The End of Dialogue in Antiquity, ed. S. Goldhill, Cambridge 2008, dokumentierten Unternehmens; vgl. jedoch den deutlich nuancierenden Einzelbeitrag von G. Clark, Can We Talk? Augustine and the Possibility of Dialogue, ebd., 117-34) bzw. eines primär von Christen betriebenen und verantworteten 'closing down' freier intellektueller Betätigung (Ch. Freeman, The Closing of the Western Mind: The Rise of Faith and the Fall of Reason, London 2002; vgl. ders. AD 381: Heretics, Pagans and the Christian State, London 2008 wo besonders der kaiserliche Beitrag zu dieser Entwicklung kritisch herausgestellt wird). Weniger radikal bemerkt eine zunehmende, nicht nur christliche Skepsis gegenüber der Dialektik und im Gefolge ein Zurücktreten der (freien theologischen) Diskussion bereits R. Lim, Public disputation, power and social order in late antiquity, The transformation of the classical heritage 23, Berkeley 1995.

${ }^{4}$ Die klassische Studie von H.J. Sieben, Die Konzilsidee der Alten Kirche. (Konziliengeschichte, Reihe B: Untersuchungen) Paderborn/München/Wien/Zürich 1979, leistet dies allenfalls im Ansatz und blendet die Fragen nach formalen Regeln völlig aus.
} 
Niklas Luhmann hat 1969 in seiner klassischen Studie mit dem Titel „Legitimation durch Verfahren“ die legitimierenden Leistungen moderner politischer Verfahren ausgeleuchtet. Er hat dabei vorgeführt, daß rationale Verfahren nicht nur ein wesentliches Instrument zur Problemdiskussion und Entscheidungsfindung darstellen, sondern gerade auch im Hinblick auf deren gesellschaftliche Akzeptanz und Wirksamwerden von Bedeutung sind. Ihre Leistungsfähigkeit ist als womöglich exakt an der kritischen Gelenkstelle zwischen Macht und Idee anzusetzen, deren abstrakte Dichotomie den Horizont vieler Konzilsstudien problematisch verengt. Luhmanns zentrale Erkenntnis ist, daß in modernen Verfahren zur Entscheidungsfindung, gleich ob in den Bereichen des Rechts, der Gesetzgebung oder der Exekutive, „richtig oder falsch“ im Kern durch soziale Interaktionsvorgänge bestimmt werden, die in politisch-administrativen Systemen institutionalisiert sind und die durch dieselben Verfahren legitimiert werden, aus denen sie hervorgehen. Beteiligte und Akteure involvieren sich selbst, indem sie bestimmte Verfahrensrollen einnehmen ${ }^{5}$, Schritt für Schritt soweit in einen Verfahrens- und Entscheidungsprozeß, daß sie schließlich dessen Ergebnis akzeptieren und mittragen müssen, auch wenn es ihren ursprünglichen Überzeugungen nur zu Teilen entspricht. Denn umgekehrt können sie sich je länger je mehr kaum noch den einmal begonnenen Verfahren entziehen, sich von dessen Ergebnissen distanzieren und Protest dagegen mobilisieren, jedenfalls nicht in einer Weise, die eine breitere Öffentlichkeit überzeugen könnte. Diese soziologische Beschreibung von Verfahren unterscheidet sich von normativen Diskussionen bei denen die Zweckrationalität und Leistungskraft von Verfahren vorrangig im Gegenüber zu einer dem Verfahren externen Norm gewertet wird. Die von Luhmann beschriebene verfahrensinhärente Legitimationsfunktion ist zugleich von anderen möglichen legitimierenden Faktoren politischer Entscheidungsprozesse wie sozialer Status, politische Autorität usw. - abgegrenzt, bzw. sie fragt spezifisch nach deren konkreter Aktivierung im Verfahren. ${ }^{6}$

Das Heranspielen der Luhmannschen Konzeption an vormoderne Gesellschaften und Denkhorizonte insgesamt, und zumal an die Synoden der Alten Kirche, ist nicht ohne

\footnotetext{
${ }^{5}$ Spezifisch zur Rollenübernahme s. Luhmann, Legitimation 82-90; vgl. 93f., 95-97.

${ }^{6}$ Für eine kritische Durchleuchtung des Luhmannschen Verfahrensbegriffs und seiner möglichen historischen Leistungskraft s. M. Sikora, Der Sinn des Verfahrens. Soziologische Deutungsangebote, in: B. Stollberg-Rilinger (ed.), Vormoderne politische Verfahren, Zeitschrift für historische Forschung, Beihefte 25, Berlin 2001, 25-51.
} 
methodische Kautelen möglich. ${ }^{7}$ Man darf speziell nicht damit rechnen, daß auf den Synoden der Alten Kirche metaphysische Gehalte schlicht nach der Art politischer Verhandlungen und Verfahren bestimmt würden. Luhmanns Beobachtungen analysieren dezidiert die nach seiner Einschätzung spezifisch modernen Merkmale solcher Prozesse in ihrer gesellschaftlichen Dimensionen und nehmen eine schon weit vorangeschrittene, und weiter zunehmende moderne Ausdifferenzierung verschiedener gesellschaftlicher Subsysteme zur Voraussetzung, die sich in autonomen Verfahren wiederum einerseits erst autopoetisch generieren, andererseits aber bereits als existierende agieren, sich perpetuieren und dabei weiter verfeinern bzw. ausdifferenzieren. Luhmanns frühe Verfahrens-Studie ist dabei erst auf dem Weg zu einer ausgeführten Systemtheorie, in der solche Systeme und ihre kommunikativen Akte im einzelnen beschrieben werden, und von der aus sich zwar theoretisch auch spätantike gesellschaftliche Interaktionen und Zustände beschreiben ließen, die aber in historischer Perspektive nicht ohne Schwierigkeiten sind und in dieser Studie nicht unkritisch zum beherrschenden kategorialen Raster gemacht werden soll. Vielmehr geht es darum, zunächst ganz grundsätzlich - und auch durch die Beobachtung von historisch bedingter Differenz zu Luhmanns Interpretation autonomer moderner Verfahren - auf die Bedeutung von Verfahren in synodalen Entscheidungsprozessen und für die Begründungsmöglichkeiten von Legitimität und Verbindlichkeit synodaler Beschlüsse aufmerksam zu werden. Dabei ist näherhin zu fragen, ob überhaupt und ggf. wie die von Luhmann freigelegten Leistungen moderner Verfahren womöglich in anderer historischer Form unter den spezifischen Bedingungen antiker kirchlich-gesellschaftlicher Kontexte auf Synoden realisiert wurden. Denn selbst und gerade wenn die im autonomen Verfahren modernen Typs geleistete Bearbeitung von Legitimitätsanforderungen und Verbindlichkeitserwartungen den Aktionsformen von altkirchlichen Synoden und dem Selbstverständnis ihrer Teilnehmer nicht entspräche - man wird vor allem die Suspendierung eines außerhalb des Verfahrens situierten Wahrheitskriteriums in den theologischen Debatten der Spätantike keinesfalls erwarten dürfen -, so bleibt gleichwohl auch in synodalen Kontexten die Problematik bestehen, Legitimität und Verbindlichkeit interagierend und kommunizierend zu etablieren und zu garantieren,

\footnotetext{
${ }^{7}$ Vgl. dazu exemplarisch etwa die Diskussion kulturhistorischer Ansätze im rechtshistorischen Kontext, B. Stollberg-Rilinger, Verfassungsgeschichte als Kulturgeschichte, Zeitschrift der SavignyStiftung für Rechtsgeschichte. Germanistische Abteilung 127 (2010) 1-32.
} 
und ergibt sich damit für den Historiker die Aufgabe, nach denjenigen Formen zu suchen, mit deren Hilfe solche Anforderungen und Bedingungen konkret zum Austrag kommen. ${ }^{8}$

Es kann hier folglich noch nicht darum gehen, Luhmanns Kategorien an den Synoden der alten Kirche im Einzelnen durchzubuchstabieren. Aus dem grob skizzierten Fragenkreis, der ein größeres Forschungsprogramm anzeigt, können vielmehr nachfolgend nur exemplarisch erste Problemstellungen im Umriß konturiert werden. Worauf es einstweilen ankommt ist, den möglichen Beitrag von Verfahrensweisen beim Zustandekommen von „Ergebnissen“ oder, in stärker formaler, soziologischer Diktion, für das Erreichen einer Reduktion von Komplexität, zu beleuchten. ${ }^{9} \mathrm{Zu}$ diesem begrenzten Zweck sollen, gleichsam im Vorfeld einer stringenten soziologischen Verfahrensanalyse zunächst einige der Rollenangebote und (impliziten) Regeln der sozialen Interaktion auf Synoden beschrieben und so den Zumutungen und Erwartungen an die Aktivitäten der handelnden Akteure nachgespürt werden, die dem Geschehen inhärent sind bzw. durch es erzeugt werden und die für die Handelnden zugleich aus der Übernahme bestimmter Rollen entstehen. Ferner gilt es nach dem Verhältnis zwischen Herstellung und Darstellung von Entscheidungen zu fragen, wobei nicht zuletzt die Frage zu beachten sein wird, wie Erwartungen und Mechanismen der Konfliktlösung im sozialen Kontext vor einer weiteren antiken kirchlichen Öffentlichkeit ausgespielt werden. ${ }^{10}$

\footnotetext{
${ }^{8}$ Angesichts der juristischen Nebentöne synodaler Entscheidungsprozesse und der unmittelbaren rechtlichen Konsequenzen von solchen Entscheidungen bei verschiedenen Gelegenheiten (siehe etwa zur Exilierung des Photin unten 000), sind dabei Luhmanns Überlegungen zu den Gerichtsverfahren von besonderem Interesse; s. Luhmann, Legitimation 57-135.

${ }^{9}$ Insoweit ist nachfolgend ein im Vergleich zu einem systemtheoretisch unterlegten Verfahrensbegriff Luhmannscher Prägung ,,weicher“ Verfahrensbegriff in Anschlag gebracht, der vor allem die spezifisch modernen Autonomievorstellungen und die Ausblendung der Möglichkeit externer Wahrheitskriterien für den antiken synodalen Kontext nicht unterstellt. S. zur Klärung und Abgrenzung des Luhmannschen Verfahrensbegriffs gegenüber anderen Konzeptionen Luhmann, Legitimation passim, insbesondere 11-26, 38-53.

${ }^{10}$ Denn nach verbreitetem theologischen Selbstverständnis der Akteure konnte es bei Synoden zur Trinitätsproblematik, ja weit grundsätzlicher bei aller menschlicher Erkenntnisbemühung in der Gottesfrage, nicht eigentlich darum gehen, Wahrheit zu „erzeugen“ - schließlich galt diese durch Offenbarung in der Heiligen Schrift als normativ vorgegeben und allenfalls exegetisch zur Sprache zu bringen - sondern ging es darum, dieser Vorgabe verstehend nachzuspüren und sie je „neu“ im Horizont der aktuellen Debatten - d.h. in der Sprache der Zeit: angesichts der Herausforderung von Häresie - zu artikulieren. „Neu“ freilich, ist darin nur der Artikulationsvorgang, nicht die vielmehr zeitlos gültige, unveränderte und unveränderliche Wahrheit. Wäre insoweit der Austrag von Dissens und Konflikt in der synodalen Praxis lediglich eindimensional als intellektuelle Aufgabe beschrieben, so ist dem für die Sicherung von „Orthodoxie“ als kirchlich-sozialer Wirklichkeit entscheidend die Notwendigkeit an die Seite zu stellen, die Verbindlichkeit solcher Erkenntnis und die Legitimität synodaler Beschlußfassungen über seine Festschreibung in propositionalen Texten und zu seiner disziplinarischen Absicherung mittels Personalentscheidungen zu etablieren, auf denen sodann auch
} 
Zwei synodale Kontexte aus der Mitte des vierten Jahrhunderts evozieren die Verquickung von Verfahrens- und Wahrheitsproblematik beim Versuch der verbindlichen Definition theologischer, Orthodoxie' in je unterschiedlicher Perspektive. In einem Fall begegnet auf den ersten Blick konventionelle theologische Diskussion vornehmlich exegetischen Charakters, wird aber in überraschender Weise institutionell situiert und funktionalisiert, so daß ihre Zielsetzung und Leistung nur aus dem Verfahrenskontext evident werden. Im anderen Beispiel wird eine auf komplizierten Wege erreichte theologische Definition letztendlich erst durch rituellsymbolisches Handeln verbindlich gemacht. Hier steht also das Verhältnis einer bereits in anderer Form sachlich fixierten und insoweit dem Geschehen vorgegeben theologischen Norm zu den Möglichkeiten und Bedingungen zur Etablierung ihrer gesellschaftlich-kirchlichen Verbindlichkeit in Frage. Die dabei zutage tretenden Handlungsweisen folgen anderen Grundsätzen und Mechanismen und repräsentieren eine andere Logik als die einer thematisch-theologischen Debatte.

$* * *$

Das erste Beispiel für in dieser Fragerichtung zu untersuchender synodaler Diskussionen und Interaktion entstammt der Auseinandersetzung mit und um die Person des Photin, eines Schülers des Markell von Ankyra, dessen Lehre er weiterführte und die als eine Form des Monarchianismus angesprochen werden kann. Photin war seit 343 Bischof von Sirmium. Bereits kurz nach seinem Amtsantritt, 343, wurde er zusammen mit Markell in der Ekthesis Machrostichos zur Zielscheibe theologischer Kritik, wenig später (345 und 347) dann zweimal als Häretiker verurteilt. Aber seinen Gegnern war es nicht gelungen, ihn aus dem Amt zu entfernen, wohl wegen beachtlichen Rückhalts in der lokalen Öffentlichkeit. ${ }^{11}$ So wurde im Jahr

gottesdienstliche Ausgestaltung und Verkündigung gründen. In dieser Perspektive wäre der Begründungshorizont „legitimer“ theologischer Entscheidungen weit über das synodale Geschehen hinaus und bis tief in die Alltagswirklichkeit christlicher Gemeinden und spätantiker, meist städtischer Öffentlichkeiten hinein auszuziehen.

${ }^{11}$ Thomas Böhm, Art. Photin von Sirmium, RGG4 6 (2003), Sp. 1322; Karl-Heinz Uthemann, Art. Photeinos v. Sirmium. LThK 38 (1999) Sp. 267; vgl. M. Simonetti, La crisi ariana nel IV secolo (StEA 11), Rom 1975, 202-206; R.P.C. Hanson, The Search for the Christian Doctrine of God, Edinburgh, 1988, 235-238, 312f. Zur Theologie s. ferner D. H. Williams: Monarchianism and Photinus of Sirmium as the Persistent Heretical Face of the Fourth Century, Harvard Theological Review 99 (2006) S. 167206. 
351, als sich Kaiser Konstantius II in Sirmium aufhielt, und auf dessen Anordnung eine Synode einberufen, um den Fall endgültig zu klären. Wie nicht anders zu erwarten, wurde Photin letztendlich abermals verurteilt und schließlich exiliert. ${ }^{12}$ Die Einzelheiten dieses Geschehens sind für die Frage nach den Verfahrensmodi für die Entscheidungsfindung höchst interessant. In Einzelheiten z.T. spannungsreiche Nachrichten darüber sind bei den Kirchenhistorikern des fünften Jahrhunderts ${ }^{13}$ und bei Epiphanius erhalten, einer bekanntlich problematischen Quelle, nicht zuletzt aufgrund der oft schlechten Textgestalt. Nach Epiphanius hatte Photin selbst um eine

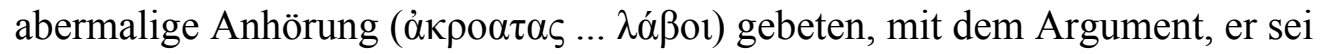
grundlos verurteilt worden. ${ }^{14}$ Diese Anhörung fand vor acht staatlich berufenen Richtern statt und wurde von sieben Stenographen aufgezeichnet. ${ }^{15}$ Epiphanius scheint Zugang zu diesen Aufzeichnungen gehabt zu haben. ${ }^{16}$ Danach fand vor den Richtern eine $\delta \iota^{\prime} \lambda \varepsilon \xi 1 \zeta$, d.h. ein Streitgespräch bzw. eine Disputation ${ }^{17}$, statt, in der Photin gegen Basilius von Ancyra antrat ${ }^{18}$, dem er schlußendlich unterlag und so

\footnotetext{
${ }^{12} \mathrm{Zu}$ den Ereignissen s. H.-C. Brennecke, Hilarius von Poitiers und die Bischofsopposition gegen Konstantius II. Untersuchungen zur dritten Phase des Arianischen Streites (337-361), PTS 26, Berlin 1984, 91-107, zum Disput mit Basilius speziell 98f.; W.A. Löhr, Die Entstehung der homöischen und homöusianischen Kirchenparteien. Studien zur Synodalgeschichte des 4. Jahrhunderts, Bonn 1986, 37 39; vgl. auch Hanson, Search 325-329, sowie knapp T. D. Barnes, Athanasius and Constantius.

Theology and Politics in the Constantinian Empire, Cambridge, MA/London, ${ }^{3} 2001,109$.

${ }^{13}$ Socr., h.e. 2.29-30 (140,9-147,2 Hansen); davon abhängig Soz., h.e. 4.6 (143,13-146,8 Bidez/Hansen), bes. 4.6.14f (145,16-146,4 Bidez/Hansen). In den Einzelheiten, zumal in der Darstellung der theologischen Positionen, vermischen beide Berichte Nachrichten von mehreren Sirmischen Synoden.

${ }^{14}$ Doc. 47,2 = Epiph., haer. 71,1,4 (250,8-10 Holl/Dummer). Wahrscheinlich bezog sich Photin mit dieser Kritik auf die angeführten früheren Verurteilungen. Es ist aber darüber hinaus auch vorstellbar, daß sein Antrag auf eine bereits bei derselben Gelegenheit unmittelbar vorausgehende abermalige Verurteilung reagiert; auf eine gewisse chronologische Unschärfe in den Etappen des Geschehens in Sirmium wird noch einzugehen sein.

${ }^{15}$ Doc. 47,2 = Epiph., haer. 71,1,5 (250,10-13 Holl/Dummer); 71,1,8 (250,24-7 Holl/Dummer). Soz., h.e. 4.6.15 (145,20-146,2 Bidez/Hansen) beschreibt das Geschehen, wenn auch sehr knapp, im Kern als Bischofsversammlung und erkennt den Beamten lediglich aufgrund kaiserlichen Mandats den Vorsitz bei dieser Veranstaltung zu. Der synodale Charakter bzw. das bestimmende Verfahrensmodell der Veranstaltungen steht aber gerade in Frage.

${ }^{16}$ Dafür spricht vor allem, daß er die Namen sowohl der Richter (Epiph., haer. 71,1,5) als auch der Stenographen $(71,1,8)$ nennen kann.

${ }^{17}$ Epiph., haer. 71,1,6 (zweimal: 250,15-17 Holl/Dummer); Soz., h.e. 4,6,14 (145,20 Bidez/Hansen);

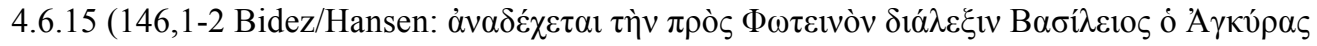

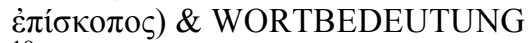

${ }^{18}$ Soz. 4.6.15 (146,1-2 Bidez/Hansen [s. vorige Anm.]), vgl. dazu die Darstellungen Epiph., haer. 71.1.6 (250,15-16 Holl/Dummer:); Socr. h.e. 2.30.42-46 (146,7-19 Hansen); Zu Basilius s. Thomas Böhm, Art. Basilius von Ancyra, LACL ${ }^{2}$ 1999, 99. W.A. Löhr, Art. Basileios v. Ankyra, LThK 2 (1994) 66-67; M. Simonetti, La crisi 259-266. BRENNECKE; Für theologischen Positionierungen in der Gruppe um Basilius, vgl. ferner Hanson, Search 349-71; W.A.Löhr, A sense of tradition: The homoousian church party, in: M.R. Barnes, D.H: Williams (Hgg.), Arianism after Arius. Essays on the development of the fourth century trinitarian conflicts, Edinburgh 1993, 81-100, bes. 87-95. L. Ayres, Nicaea and its Legacy. An Approach to Fourth-Century Trinitarian Theology, Oxford 2004, beschreibt in einer knappen Skizze der Synode Basilius als 'main accuser' (134) des Photin, unterstellt also ein im
} 
abgesetzt und ins Exil geschickt wurde. Durch die Bestellung von staatlichen Beamten als Richtern sowie die Beteiligung von weiteren Notabeln ${ }^{19}$ ähnelt das Geschehen einem Zivilprozeß oder einer Verwaltungsanhörung, also juristischen Formaten, und ist insoweit nach Form, Situierung und vor allem Rollenzuschreibung der Beteiligten von synodalen Verfahren allein unter Bischöfen unterschieden. ${ }^{20}$ Die Beteiligung eines erweiterten Kreises von Vertretern der sozialen Eliten stellt zugleich einen ersten deutlichen Bezug zu einer über- bzw. außerkirchlichen Öffentlichkeit her. Gleichzeitig nimmt es Elemente von philosopisch-rhetorischen Debatten auf, die im öffentlichen Raum und in den Bildungsstädten der antiken Stadt vielfältig anzutreffen waren und die sich beispielhaft noch die durch Auftritte des Augustinus gegen verschiedene Manichäer illustrieren lassen. Sie unterstreichen den sozialen Konnex und evozieren die Konventionen des intellektuellen Wettstreits. ${ }^{21}$ Eine genauere Bestimmung der Verfahrensformen und ihres synodalen Charakters wird durch eine chronologische Unklarheit verkompliziert. Nach den Darstellungen der Kirchenhistoriker folgt die Debatte zwischen Basilius und Photin auf einen vorausgehenden synodalen Beschluß und somit in der Verlängerung, aber auch Verlagerung, der Aktionsweisen der synodalen Versammlung. Ursprünglich habe die Synode Photin wegen der Lehren des Sabellius und Paul von Samosata verurteilt, ihm dann aber die Möglichkeit zur Versöhnung angeboten. ${ }^{22}$ Nach Sokrates, dem

Kern rechtliches Format. Dies wird der Disputation zwischen beiden nur insoweit gerecht, als sie rechtliche Konsequenzen zeitigt, beschreibt aber die Rollenverteilung beider Kontrahenten nicht völlig zutreffend.

${ }^{19}$ Nach Socr., h.e. 2.30 .43 (146,12 Hansen) handelt es sich um (im comitatus des Konstantius

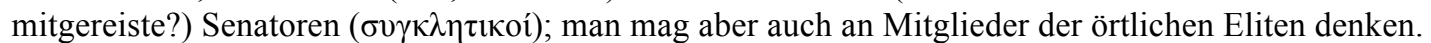
Während er so deren sozialen Rang als entscheidend für ihre Beauftragung herausstellt, ist das Auswahlkriterien bei Soz., h.e. 4.6.15 (145,21-146,1 Bidez/Hansen), ihre Bildung und Erfahrung mit rhetorischen Wettstreiten. Zu den namentliche genannten Richtern, s. Brennecke, Hilarius 96f., Anm. 22.

${ }^{20}$ T.D. Barnes, Athanasius and Constantius 109 will dieses Verfahrenselement von der ,eigentlichen Synode (the council proper) unterschieden wissen und situiert es als separates Geschehen im Frühjahr, die „Synode“ hingegen im Herbst 351. Er bestreitet zudem ein Richteramt der Senatoren - entgegen

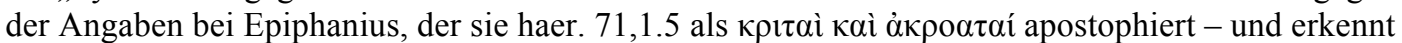
ihnen lediglich die Aufgabe zu, die Verläßlichkeit der Protokollierung zu garantieren. Dabei versteht er die Geschehnisse als „Voruntersuchung“ (preliminary investigation). Zur weiteren Beschäftigung mit Barnes Rekonstruktion, vgl. unten, Anm. 000 [22. 26f.].

${ }^{21}$ S. Augustinus, Contra Fortunatum; vgl. einführend V. H. Drecoll, M. Kudella, Augustin und der Manichäismus, Tübingen 2011, 134-138; dagegen anders im Charakter und mit deutlichen rechtlichen Konsequenzen: Contra Felicem (vgl. ebd. 138-144). Vgl. allgemein Lim, Public disputations, 70-108; und im philosophisch-schulischen Kontext 31-69.

${ }^{22}$ Socr., h.e. 2.30.42 = Doc 47.2 (146,7-10 Hansen). Auch nach Soz., h.e. 4.6.14 wird ihm die Rückgabe des Bistums in Aussicht gestellt, doch hatte Photin seinen Sitz noch nicht verlassen. Die etwas unklare Formulierung bei Epiphasius situiert ebenfalls das Geschehen ,nach' ( $\mu \varepsilon \tau \alpha$ ) seiner Verteidigung in Serdika. Meinte Epiphanius damit einen Auftritt vor der Synode, der dem Ersuchen um eine Anhörung vorausging? Dann wäre die von ihm als grundlos betrachtete Verurteilung wohl nicht 
Sozomenos wiederum folgt, war die Debatte zwischen beiden dann die Folge des gescheiterten Versuchs der Synode, sich mit Photin zu versöhnen, und stellt damit eine Ergänzung und Fortentwicklung des Synodalgeschehens im engeren Sinne dar. Die Bischöfe hatten Photin zunächst aufgefordert, eine gemeinsame Erklärung zu unterzeichnen. ${ }^{23}$ Dies ist der synodale Normalfall, um Konsens zu erzielen und mit der geleisteten Unterschrift zugleich darzustellen. Nun hingegen substituierte ein anderes Verfahren für dieses Ziel. Denn Photin hatte diesen Vorschlag abgelehnt und statt dessen zu einer Disputation herausgefordert, die auch auf kaiserliche Anordnung zustande kam. Die Kirchenhistoriker berichtet weiter, daß diese vor den genannten staatlichen Richtern und Notabeln und im Beisein der Bischöfe geschah, wobei Basilius von Ankyra die Rolle des Disputanten übernahm. ${ }^{24}$ Durch die Anwesenheit der Bischöfe ist der Konnex mit der vorherigen Synodalversammlung offenkundig. Gleichwohl ist ihre Rolle fundamental verwandelt: aufgrund der bestellten staatlichen Richter sind sie nun praktisch in die Rolle der Zuhörer bzw. des Publikums gedrängt, während sie nach synodalem Brauch als gleichberechtigte Richter und ggf.

Diskutanten agierten. Auch die Rolle des Disputanten wird demgegenüber hier nur noch allein von Basilius stellvertretend wahrgenommen. Man wird eine Beauftragung durch die Synode annehmen dürfen; in jedem Fall repräsentierte Basilius den von den Bischöfen zuvor bereits gemeinschaftlich eingenommenen anti-photinianischen Standpunkt und damit mindestens de facto auch den kollektiven Willen und die „gemeinsame“ Theologie der Synode. Die in Aussicht genommenen Debatte überträgt und verschiebt also die Beratungen und theologischen Reflexionen der Synode in ein

eines der früheren Urteile - Epiphanius spricht zuvor irrtümlich von einer Verurteilung in Serdika -, sondern der aktuelle Beschluß der sirmischen Synode. Im Anschluss an die Darstellung der Kirchenhistoriker rechnet Brennecke, Hilarius 96f. mit einer voraufgehenden synodalen Versammlung; Löhr, Entstehung 180, Anm. 222 dagegen ausschließlich mit dem Abhalten einer Disputation. Will man angesichts der unstreitigen Konfusion des Sokrates im übrigen seiner Chronologie kein Vertrauen schenken, und seine Ansetzung einer „Synode“ vor der Disputation für eine Fiktion oder ein Mißverständnis halten, so wird die hier vorgetragene Deutung nicht im Grundsatz berührt. Worauf es ankommt, ist die Verwandlung von Verfahrenselementen in den verschiedenen Schritten eines komplexen Ablaufs. Vgl. aber unten, Anm. 000.

$\mathrm{Zu}$ einer anderen Chronologie gelangt Barnes, Athanasius and Constantius 109 (s.o. Anm. 000[20]). Seine Vorstellung einer nach der „Voruntersuchung“ erst im Herbst zu versammelnden Synode scheint

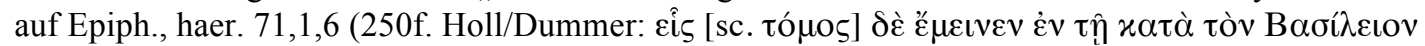
бvvó $\omega$ zurückzugehen; er paraphrasiert ,... one [sc. copy of the minutes] for its use by the council of bishops destined to decide whether the theology of Photinus was orthodox or heretical...". Allerdings kann Epiphanius den terminus synodos gelegentlich untechnisch und eher im Sinne von Gruppierung oder Partei verwenden, was mir an der vorliegenden Stelle plausibel erscheint; vgl. haer. 73, 23,3; $73,27,5 ; 73,28,3 ; 73,35,3 ; 73,37,4$ - bei einer Reihe weiterer Stellen ist die institutionelle Bedeutung von synodos mindestens unscharf. Ich danke Herrn Prof. Löhr für den Hinweis auf diesen Umstand.

${ }^{23}$ Soz., h.e. 4.6.14 (145,17-19 Bidez/Hansen $)=$ Doc. 47.2.

${ }^{24}$ Soz., h.e. 4.6.15 (145,20-146,2 Bidez/Hansen). 
anderes Forum und Format und instrumentalisiert sie zugleich für eine neue Zwecksetzung. Denn prinzipiell ist zwar auch weiterhin vorstellbar, daß die Debatte Photin dazu bringen sollte, die zuvor zurückgewiesene Erklärung doch noch zu unterzeichnen. Damit wäre die ursprüngliche Absicht der Synode realisiert. Selbst in dieser Blickrichtung aber, so bleibt festzuhalten, hatte die Debatte für die Synode gerade nicht die Aufgabe, Glaubensinhalte so zu erörtern, daß es zu einer neuen Festschreibung von Orthodoxie käme - denn darauf hatte sich die Synode mit ihrer Erklärung ja bereits verständigt. Vielmehr konnte es nur um das Bemühen gehen, Photin zu überzeugen, dem bestehenden Konsens beizutreten.

Realistischer aber mußte wohl eine veränderte Zielsetzung im Vordergrund stehen, nämlich ihn gegenüber den staatlichen Richtern als unzweifelhaft häretisch zu überführen und damit den Kaiser zu veranlassen, ihn aus dem Amt zu entfernen und ins Exil zu schicken. Mit dieser wahrscheinlicheren Zielsetzung wird das ursprüngliche Anliegen der Synode ebenso wie der Verfahrenshintergrund nochmals in Teilen transformiert. In der eins-zu-eins Debatte handelt die Synode nicht mehr kollektiv, sondern nur noch durch einen rhetorisch und theologisch ausgewiesenen Repräsentanten. Gleichzeitig aber agiert dieser wie ein Interessenvertreter oder Anwalt für den (Verfahrens-) Antrag, Photin von seinem Sitz zu entfernen. Ein solches Ersuchen blieb wahrscheinlich unausgesprochen und war jedenfalls nicht soweit unsere Quellen berichten - juristisch formalisiert. Aber ein Urteilsspruch der staatlichen Richter konnte von vornherein nur in einer Entscheidung über Photins Verbleiben im Amt oder seiner Entfernung aus dem Amt bestehen. Interessant ist aber, daß eine solche Entscheidung mindestens indirekt zugleich als Urteil über der Sachstand der Orthodoxie verstanden werden konnte, vielleicht sogar mußte. Deutlich wird dies nicht zuletzt, wenn man das Verfahren aus der Perspektive des Photin zu verstehen sucht. Sein Ziel muß es gewesen sein, aus dem Konflikt siegreich hervorzugehen, d.h. von den Richtern als überzeugend oder zumindest als in seinen Ansichten und Argumenten nicht widerlegt bewertet zu werden. Damit wären vorherige Synodalurteile abermals ins Leere gelaufen und hätte er seinen Bischofssitz weiter behalten können. Die Bereitschaft der Synode, sich auf ein solches Prozedere mit offenem Ausgang einzulassen, zeigt entweder ihre unerschütterliche Zuversicht in ihre Sache oder verdankt sich der zähneknirschenden Einsicht in die fehlende Durchsetzbarkeit ihres Urteils, so daß sogar die Möglichkeit einer „Niederlage“ und damit des Amtsverbleibs des Photin hingenommen werden mußte. Mindestens für 
Photin, und wohl auch für die staatlichen Stellen, mußte ein solches Urteil zugleich eine Anerkenntnis der Stichhaltigkeit oder gar Überlegenheit seiner theologischen Position mitenthalten, selbst wenn keine formale Entscheidung über „den Glauben“ erwartet werden konnte, und dies nicht im offiziellen Auftrag der Anhörung liegen konnte. Umgekehrt war die Feststellung und propositionale Fixierung „orthodoxer“ Theologie in der zuvor erarbeiteten Erklärung unwirksam, solange sie nicht durch Unterschriftsleistung verbindlich anerkannt war, bzw. bis daß ihr Standpunkt, überführt in die Modi rhetorischer Debattierkunst, nicht Überzeugungskraft vor den staatlichen Richtern gewonnen hatte.

Die vorgelegte Analyse der Abfolge unterschiedlicher Verfahrensschritte und der Auswirkungen der dabei zur Anwendung gebrachten Verfahrensformen auf die Ziele der Entscheidungsfindung, die Rollen und Aktionsmöglichkeiten der Beteiligten und auf die Verquickung von theologischer Sachklärung und juristisch-administrativer Beschlußfassung ruht auf der Chronologie der Berichte der spätantiken Kirchenhistoriker. Da sich aber Echos der Debatte in den Synodalbeschlüssen wiederfinden, schlagen die Editoren der Dokumente eine andere Abfolge vor und verstehen die Debatte als Teil der Synode ${ }^{25}$ - wobei allerdings eine mögliche Verfahrensbeteiligung der Beamten und Notabeln über die Beurteilung der Debatte hinaus auch bei den theologischen Beschlüssen, d.h. bei der Erstellung und Verabschiedung der sog. „Ersten“ sirmischen Formel, nicht diskutiert wird. ${ }^{26}$ In einer

\footnotetext{
${ }^{25}$ Brennecke/Stockhausen etc [Hgg.], Dokumente 000; so im Kern bereits Löhr, Entstehung 180, Anm. 222 (s.o. Anm. 000). Bei einer solchen Rekonstruktion bleibt aber das Zustandekommen der Anathematismen unerklärt. Es muss in jedem Fall (mindestens) eine Fortsetzung des Debattengeschehens in einem anderen Verlaufsmodus, vielleicht sogar eine weitere Zusammenkunft gegeben haben, um diese Anathematismen zu formulieren und zu beschließen. Vgl. ferner folgende Anm.

${ }^{26}$ Die Funktion der Formel als Dokument einer möglichen Versöhnung mit Photin, bzw. als desjenigen Standards, an dem er seine Orthodoxie zu beweisen hatte, wie ihn die Kirchenhistoriker beschreiben, wäre in dieser Rekonstruktion aufzugeben; welchen Zweck die Formel erfüllte, bliebe unbeantwortet. Womöglich ist die Spannung zwischen der Chronologie der Berichte und den Beobachtungen zu den weiteren Dokumenten aber lösbar. Abgesehen von den Notizen über eine Debatte sind von der Synode eine Glaubenserklärung und 27 Anathematismen überliefert. Wenn die Historiker des 5. Jh. die Reihenfolge annähernd korrekt wiedergeben, wäre die Glaubensformel vor der Debatte verfaßt und stellte damit jenen Text dar, der Photin zur Unterschrift präsentiert wurde. Die angehängten Anathematismen hingegen spiegeln in ihrem exegetischen Charakter, d.h. der Zurückweisung spezifischer Mißverständnisse und Fehlinterpretationen einzelner Schriftstellen, mindestens in Teilen die Diskussion zwischen Basilius und Photin. Sie illustrieren jedenfalls den Charakter des exegetischhermeneutischen Konfrontation, in die beide sich verwickeln (Berührungen beobachtet bereits J.N.D. Kelly, Early Christian Creeds, 3. Aufl. London 1972, 281; vgl. Löhr, Entstehung [wie vorige Anm.], Brennecke/Stockhausen etc [Hgg.], Dokumente 000. Ein unauflöslicher Widerspruch muß daraus jedoch nicht notwendig resultieren. Vielmehr wäre die Verknüpfung von unterschiedlichen Verfahrenschritten mit jeweils einhergehendem Wechsel der Verfahrensform und des Forums zwischen kirchlichen und kaiserlich-gesellschaftlich bestimmten institutionellen Rahmungen und Modi
} 
solchen Rekonstruktion hätte der Kaiser der Synode in Gestalt einer stellvertretend geführten Debatte die Verfahrensform vorgeschrieben, die Entscheidungsstellen mit seinem, nicht-kirchlichen, Personal besetzt und ihr damit die Entscheidungskompetenz faktisch aus der Hand genommen. Das ist nicht prinzipiell undenkbar. ${ }^{27}$ Auch eine solche Abfolge kreiert eine komplexe Verwebung unterschiedlicher Formelemente und Verfahrenserwartungen, wobei auch in diesem Fall die Synodalen, nun womöglich in Beugung vor staatlicher Macht, sich auf ein Verfahren einlassen (mußten), bei dem sie riskierten, mit ihren Absichten nicht zum Zuge zu kommen. Gleichwohl wäre auch nach dieser Rekonstruktion die Entscheidung nicht vollständig in den Bereich staatlicher Rechtsfindung

womöglich lediglich um eine weitere Wendung zu erweitern. So könnten durchaus zunächst die Glaubensformel von der Synode verabschiedet, Photin zum Zweck der Rekonziliation vorgelegt und er wegen fehlender Bereitschaft zur Unterzeichnung (abermals) verurteilt worden sein - Bezieht sich Photins Initiative hingegen auf die früheren Verurteilungen, so könnte alternativ eine bereits existierende Formel benutzt worden sein, so daß keine ausführliche synodale Versammlung vor der Debatte vonnöten wäre. Eine wie auch immer geartete vorbereitende Zusammenkunft der Photingegner, auf der eine geeignete Formel identifiziert, Photin entgegengehalten und diesbezügliche Vorabsprachen getroffen worden wären, ist aber auch dann anzunehmen. -Nach der Durchführung der von Photin geforderten Anhörung in Gestalt der Debatte mit Basilius hätte die Synode in (nochmaliger?) Zusammenkunft, und in der Reaktion auf die Debatte, die Anathematismen verfaßt oder mindestens bearbeitet und ergänzt. Auch Brennecke, Hilarius 100 rechnet mit einem solchen Schritt nach der Disputation. Somit hätten wir es zwar mit einem großen Ereignis in direktem zeitlichen Zusammenhang zu tun, das allerdings analytisch und im Verfahrensablauf in mehrere Einzelschritte zu untergliedern wäre, deren je spezifischer Charakter sich in der Interaktion von kirchlichen und gesellschaftlichen Verfahrensangeboten ausarbeitete.

In Barnes' Rekonstruktion einer Abfolge von Voruntersuchung und eigentlicher Synode (Athanasius and Constantius, 109) wäre dieses Geschehen zeitlich sehr gedehnt bzw. in zwei separate Ereignisse zerlegt. Das Entstehen der Formel ebenso wie das der Anathematismen wäre in der ,eigentlichen Synode“" anzusiedeln, und beide als eine synodale Weiterführung in Reaktion auf das Resultat der Debatte zu verstehen. Auch damit wäre wenigstens ein Verfahrenswechsel angezeigt; unklar bliege dann allerdings, anhand welchen Maßstabs Photin ,voruntersucht“ wurde.

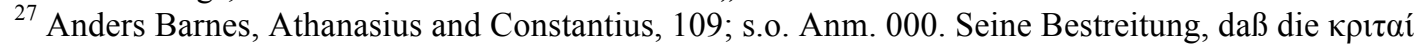
des Epiphanius eben nicht Richter sein dürfen, ist nicht eingehend begründet. Sie scheint auf einer grundsätzlichen Erwägung zu beruhen, wonach seit Konstantin kein Kaiser oder kaiserlicher Vertreter direkt über Bischöfe richtete (vgl. ebd. 172f.). Historisch gesehen ist es jedoch gerade Konstantius, der in den Abläufen der Jahre 359/60 einer Verletzung des Ideals der Nichteinmischung wenigstens nahekommt. Womöglich ist seine Beautragung der Notabeln in Sirmium 351 ein Schritt auf dem Wege zu einer solchen, robusteren Haltung. Wenn die hier präferierte Interpretation richtig ist, wonach die staatlichen Richter auf ein existierendes Synodalurteil zurückgreifen konnten und lediglich seine Validität auf Photins Einspruch hin überprüften, wird auch Barnes' prinzipieller Anstoß vermieden. Barnes' übergeordnete, an sich - jedenfalls in ganz überwiegendem Maß der Fälle - richtige Beobachtung zur Abgrenzung von staatlichem und kirchlichem Handeln macht aber vor allem auf ein interpretatorisches Dilemma aufmerksam, wenn man das Geschehen aus einer polaren Gegenüberstellung von kaiserlicher Gerichtsbarkeit und synodaler Entscheidungsgewalt als von zwei mehr oder minder klar vorgegebenen Größen deuten will. Diese Studie will vielmehr auf die Verwebung beider - und weiterer - Dimensionen kirchlich-gesellschaftlichen Diskurs- und Entscheidungshandelns aufmerksam machen, die handelnd je neu austariert werden und auf diese Weise legitime Verfahren und institutionelle Formen erst schafften. An den Schnittstellen möglicherweise konkurrierender Erwartungen und potentiell unterschiedlicher institutioneller Orientierungen und Vorbilder definieren sich die Situation und die Rollen der Beteiligten im Vollzug des konkreten praktisch geübten Verfahrens. 
hinübergezogen, waren doch Gegenstand und Maßstab der Beurteilung theologischer Natur, und bediente sich die Klärung auch theologischer Argumentationsformen, wie die Debatte im einzelnen zeigt.

Im zweiten Schritt bedarf darum die fragliche Debatte selbst der Analyse. ${ }^{28}$ Für ihren Charakter und Ablauf ist schon die von Epiphanius, Sokrates und Sozomenus gebrauchte Terminologie aufschlußreich. Epiphanius rechnet mit Fragen ( $\pi \varepsilon v ́ \sigma \varepsilon ı \varsigma)$ des Photin und erwartet, daß Basilius diese seinerseits hinterfragt und dagegen

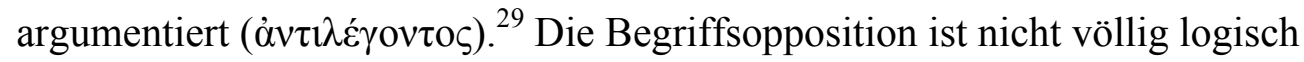
stringent, aber in der Sache klar. Sozomenos stellt konventioneller Fragen und

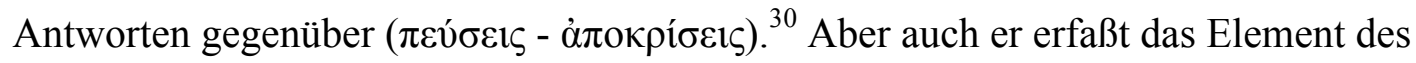

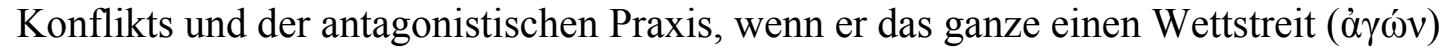
nennt. ${ }^{31}$ Sokrates spricht in dieser Hinsicht besonders deutlich sogar von einem Kampf der Worte bzw. Reden ( $\mu \alpha ́ \chi\rceil \ldots . . . \lambda$ ó $\omega \omega v) .{ }^{32}$ Die etwas schiefe Gegenüberstellung bei Epiphanius lenkt unsere Aufmerksamkeit also auf zwei Zutaten des Debattenformats, die auch in den Vorstellungen der späteren Historiker gegenwärtig sind: das intellektuelle Bemühen um Sachklärung in Frage und Antwort und auf den streitigen Austrag der dabei zutage tretenden Differenzen in Gestalt des Rededuells. Dabei ist das $\dot{\alpha} v \tau \imath \lambda \varepsilon ́ \gamma o \mu \alpha^{33}$ in dieser Konstellation nicht ein Format, das

\footnotetext{
${ }^{28} \mathrm{Zu}$ dieser Debatte s. Brennecke, Hilarius 94f.; Löhr, Entstehung 180, Anm. 225.

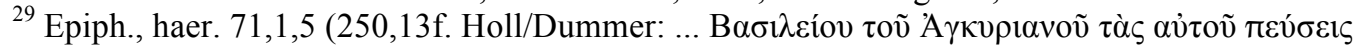

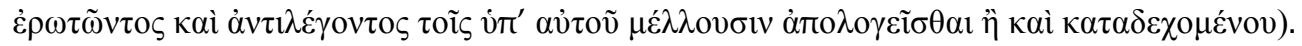

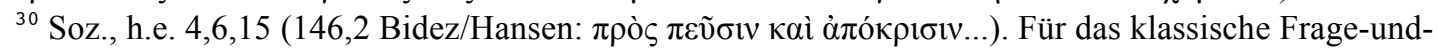
Antwort Format, das der Bearbeitung von intellektuellen Problemen aller Art dienen kann, vgl. H. Dörrie, Dörries H., Art. Erotapokriseis, RAC 6 (1966) 342-70; sowie für die entsprechende Literatur Yannis Papadoyannakis, Instruction by Question and Answer: The Case of Late Antique and Byzantine Erotapokriseis, in: Scott F. Johnson (ed.), Greek Literature in Late Antiquity: Dynamism, Didacticism, Classicism, Aldershot 2006, 91-105. Seine spezielle Zuspitzung erfährt das Format hier durch den streitigen Charakter der Debatte.

${ }^{31}$ Soz., h.e. 4.6.15 (146,3 Bidez/Hansen).

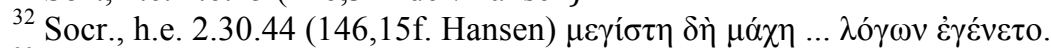

${ }^{33}$ Der Begriff und das zugehörige Wortfeld als solche geben zwar an sich noch nicht notwendigerweise Anlaß, das Gegeneinander der Redner und den Gegensatz der Argumente als primär polemisch orientiert und Ausdruck unversöhnlicher Opposition zu verstehen (s. Diccionario Griego-Español, Bd.

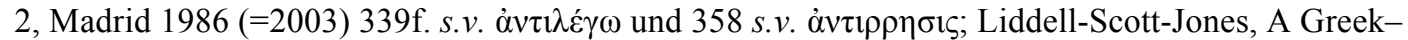

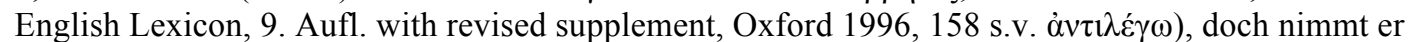
verschiedentlich solche Nebentöne von scharfer Kontroverse und durchaus auch persönlich herabsetzenden Angriffen auf. Diese Dimension illustriert anschaulich die Definition der Antirrhesis bei Henry Peacham, The Garden of Eloquence (1593) (repr. Delmar, New York, Scholars' Facsimiles \& Reprints 1977); er schreibt, s.v. antirrhesis: Antirrhesis is a forme of speech by which the Orator rejecteth the authority, opinion or sentence of some person: for the error or wickednesse in it ... This forme of speech doth specially belong to confutation and is most apt to refell errors and heretics, and to reject evil counsell and lewd perswasions. Diese Schärfe schwingt sicher auch schon in Eccl. 8.1 und 4Mak 6.1 mit. In der Kontroverse zwischen Basilius und Photin jedenfalls scheint mir der polemisch-
} 
eine freundschaftliche und letztlich konsensuelle Konflikt- und Problemlösung befördert; es läßt vielmehr Sieger und Besiegte erwarten. Diese Notwendigkeit ist im Gefälle konventionellen Denkens der Zeit und nicht nur eines Epiphanius unproblematisch: danach werden Häresie und Orthodoxie als konfligierende Kräfte verstanden, die in einem Kampf von Gut und Böse von letztgültiger, eschatologischer Bedeutung und apokalyptischer Proportion miteinander verstrickt sind. In dieser Blickrichtung ist kein Raum für Vorstellungen einer gemeinschaftlich-diskursiven Suche nach einem angemessenen Ausdruck und Verständnis der Trinitätsproblematik. In Epiphanius Perspektive, vom Standpunkt „seiner“ Orthodoxie aus gesehen, bedürfen darum die Fragen des Photin weniger einer Antwort als vielmehr der nachdrücklichen Gegenrede.

Das streitige Format der Debatte kann also - in historischer Perspektive - kaum geeignet gewesen sein, noch war es dazu gedacht, Konsens herbeizuführen oder tiefschürfende konzeptionelle Klärung zu befördern, zumal in der beschriebenen Konstellation die Resonanz in der (allerdings begrenzten) Öffentlichkeit, außerhalb des eigenen Kreises des Bischofskollegiums, eine (vor-) entscheidende Bedeutung gewinnt. Es geht mithin nicht um eine abstrakte Rationalität der Standpunkte, sondern um deren Überzeugungskraft in der konkreten Situation rhetorischer Umsetzung. Die Debatte teilt insoweit den agonalen Charakter klassischer Rednerduelle und erlaubt es nicht, argumentative Substanz und polemisch-agitatorische Umsetzung artifiziell zu unterscheiden.

Für die Auffassung der im Geschehen zu klärenden Problematik bedeutet dies zugleich, daß sie nicht als primär intellektuell zu erfassende theologische Schwierigkeit einer erst herzustellenden Lösung zugeführt werden soll, sondern der rhetorischen Demonstration einer in der Sache klaren, vorentschiedenen Antwort bedarf, die weniger den Debattengegner als die bestellten Richter von ihrer Überlegenheit überzeugen muß.

Epiphanius berichtet weiter, daß Photin damit geprahlt habe, hundert Schriftbeweise ( $\mu \alpha \rho \tau v \rho i ́ \alpha$ ) für seine Ansichten beibringen zu können. ${ }^{34}$ Wo Epiphanius nachfolgend direkte Rede benutzt und sich dabei womöglich auf das Protokoll bezieht - natürlich wird man nicht völlig ausschließen können, daß die Debattenausschnitte zum Teil 
eher „nachempfunden“ als aus den Protokoll zitiert sind - stehen denn auch tatsächliche ganz unmittelbar einzelne Schriftstellen und -passagen und deren Interpretation für die Trinität und Christologie im Zentrum der Diskussion. Innerhalb des Debattenformats und quasi-gerichtlichen Situationsrahmens wird also durchaus in einer Weise argumentiert, die man von theologischer Sacherörterung erwarten möchte. Gleichzeitig wird das Scheitern dieser Strategie offenkundig. Denn, so Epiphanius, auf die Nachfragen des Basilius ,wie“ die Schrift bestimmte Vorstellungen lehre, akzeptierte Photin jeweils die Valenz des Texts, führte aber hermeneutische Unterscheidungen ein, die ihn zu einem anderen Verständnis führten. ${ }^{35}$ Die hundert Schriftzeugnisse des Photin und die entsprechenden Texte des Basilius, also das schiere Volumen der Beweisstellen, führte mithin nicht zu einem Ergebnis der Debatte, und erst recht nicht zur theologischen Sachklärung, weil ihr konzeptionelles Verständnis an unterschiedlichen Vorentscheidungen hermeneutisch auseinanderbrach.

Für das Wesen und die Formelemente der zwischen Basilius und Photin geführten Debatte können so das Frage-und-Antwort-Format, ein deutliches Element der Konfrontation und des Streits, die Erwartung von Sieg und Niederlage und eine klare inhaltlich-methodische Grundlegung in der Auslegung der Schrift als bestimmende Merkmale identifiziert werden. Gleich ob man die so geführte Debatte den Kirchenhistorikern folgend chronologisch nach einem vorausgehenden Synodalbeschluß verortet oder sie innerhalb des synodalen Ablaufs plaziert, bleibt festzuhalten, daß sie sich als Extension und teilweise Transformation der Synode in einen benachbarten gesellschaftlich-verwaltungsjuristischen Raum darstellt und nicht $\mathrm{zu}$ theologischen, sondern rechtlichen Ergebnissen führen soll sowie auf öffentlichkeitswirksame Demonstration von Überlegenheit setzt. Sie ist damit zugleich ein Beispiel für das komplexe Miteinander und Ineinander von synodalkirchlichen und staatlichen Interessen in den Konflikten des vierten Jahrhunderts generell. Dieses Verwebungen und Spannungen, so zeigt sich, tragen sich nicht zuletzt in den zur Anwendung gebrachten entsprechenden Verfahrensformen und -modellen aus. Als polemisch-antagonistische, exegetisch fundierte Debatte zur quasijuristischen Entscheidungsfindung vor staatlichen Richtern und mit den soeben noch als Synode konstituierten Bischöfen nunmehr in der Rolle des Publikums stellt das

\footnotetext{
${ }^{35}$ Epiph., haer. 71.2.1-3 (251,3-16 Holl/Dummer).
} 
Geschen eine polyvalente Veranstaltung dar, in der sich verschiedenen Interessen und Zwecksetzungen überkreuzen. Im Spannungsfeld unterschiedlicher Erwartungen an ihre Leistungsfähigkeit, sei es als theologische Diskursstrategie zur im Kern exegetischen Beantwortung der Trinitätsproblematik, sei es als rhetorischperformative „Aufführung“ mit dem Ziel der öffentlich sichtbaren und sozial wirksamen Überwindung und Bloßstellung des Gegners, sei es als Prozeßstrategie zur Überführung des Gegenübers als schuldhaft (im Sinne eines unausgesprochen bleibenden, aber vorauszusetzenden Maßstabs, hier: von Orthodoxie) und Erwirken des (Verbannungs-)Urteils, ist die Debatte auf der Schwelle verschiedener intellektueller und institutioneller Anforderungen und Erwartungen höchst prekär situiert.

$* * *$

Komplexe Verwicklungen vielgestaltiger Verfahrensabläufe und -modalitäten zeigen auch das Doppel-Konzil von Rimini und Seleukia und seine sorgfältige Vorbereitung. Es war als Höhepunkt und Abschluß der theologischen Diskussionen und der kaiserlichen Religionspolitik der 350er Jahre geplant und sollte die Wiederherstellung der kirchlichen Einheit erzielen. Auch bei dieser Veranstaltung wird nicht einfach auf diskursivem Wege in kühler Rationalität der Versuch einer theologische Sachklärung betrieben, noch genügt der Hinweis auf kaiserliche Machtausübung zur Erklärung seines Resultats. Im Spannungsfeld theologischer Meinungsunterschiede und politischer Druckkulisse wird gerade hier deutlich, welch große Bedeutung den Handlungsweisen der Teilnehmer zukommt, um dem schließlich erzieltem Übereinkommen Plausibilität und Legitimität zuzuschreiben und zu sichern. Dabei erweisen sich einzelne Aktivitäten der Synode als hochgradig inszeniert und zeigen mindestens in Teilen - rituell-symbolischen Charakter. Ihnen kommt die Aufgabe zu, nicht erst eine thematisch-sachliche Übereinstimmung herbeizuführen, sondern einen bereits mit anderen Methoden erzielten Konsens im Blick auf eine weitere Öffentlichkeit darzustellen. In der nachfolgenden Analyse soll es wesentlich um diese Dimension des Geschehens gehen, ohne daß die vielschichtigen Fragen der theologischen Positionsbestimmung der handelnden Parteien und der Wirkungen 
kaiserlicher religionspolitischer Aktivität nochmals im einzelnen aufgegriffen werden sollen. ${ }^{36}$

Die Vorbereitung der Synode beginnt bereits mit einem als Vorbereitungstreffen zu bewertenden Zusammenkunft einer kleinen Gruppe von Bischöfen in Sirmium, die das von den Gegnern vielfach verspottete und verhaßte sog. Datierte Bekenntnis (22 Mai 359) entwarfen. ${ }^{37}$ Im Rückblick auf die Ereignisse und zur eigenen Rechtfertigung als Beteiligter betont bereits ein Bericht des Ortsbischof Germinius die Regelhaftigkeit der Art und Weise, in der dieses Bekenntnis als Resultat von ausführlichen Diskussionen zustande kam. ${ }^{38}$ Auch wenn die dabei unterstellten Kriterien in der Sache unanschaulich bleiben, ist die Tatsache als solche wichtig, daß Germinius ein Verfahren vor Augen führen will, das robust und regelkonform war und den zu erwartenden Standards entsprach, und darum weder als ungeordnetes Durcheinander verstanden werden dürfe noch als ein Geschehen, das sich fremden Einflüssen beugte. Es ist also die Regelhaftigkeit des Vorgehens, das die Bedeutung und Geltung des aufgrund dieser Diskussion entstandenen Positionspapiers (die fides) sicherstellen soll.

Das Treffen der Vorbereitungsgruppe zeigt ferner an, daß die große Synode sorgfältig geplant wurde, um das angestrebte Ziel, den Riß zwischen Ost und West zu heilen, zu erreichen. Planung und ein komplexes Ineinander verschiedener Verfahrensformen und Schritte prägen denn auch in einer elaborierten Choreographie den Ablauf der Einigungsversuche zwischen den beiden Synoden und dem Kaiser. Konstantius lud zu zwei separaten Synoden der Bischöfe beider Reichsteile, verlangte, daß sie ihre Theologie niederlegten, und zwar in einer Weise - soviel ist klar - wie es dem Text der Vorbereitungsgruppe entsprach; sodann sollten beide Seiten Delegationen von je zehn Bischöfen zum Hof schicken, die dort die endgültige Einigung aushandeln und formalisieren sollten, die dann wiederum zurück vor die jeweilige Synode gebracht

\footnotetext{
${ }^{36}$ S. grundlegend Brennecke, Homöer 5-46, für die Ereignisse in Rimini speziell 5-40.

${ }^{37}$ Doc. 56.2, überliefert bei Stelle Athan., syn. 8.3-7 (235,21-236,15 Opitz); Socr., h.e. 2.37.18-24 (154,6-155,9 Hansen). Vgl. zur Theologie der Formel Löhr, Entstehung 99-102; Brennecke, Homöer $16-23$.

${ }^{38}$ Hil., coll.antiar. B $6.3(163,17-22$ Feder $)=$ Doc. 56.1 . ... post habitam usque in noctem de fide disputationem et ad certam regulam perductam Marcum ab omnibus nobis electum fidem dictasse, in qua fide sic conscriptum est ... (,nach einer bis in die Nacht dauernden und nach einer festen Regel durchgeführten disputatio über den Glauben diktierte der von uns allen dazu ausgewählte Marcus [von Arethusa] den Glauben; in diesem Glauben[stext] ist folgendes geschrieben ... [es folgt ein Zitat aus der fides]").
} 
und ihr zur Unterschrift vorgelegt werden sollte. ${ }^{39}$ Seine entsprechenden Anweisungen legen präzise die erwarteten Schritte, die Aufgabe der Synode(n) und die Grenze ihrer Kompetenzen fest. ${ }^{40}$ So entsteht eine komplizierte Choreographie von synodaler Diskussion, diplomatischer Mission und Verhandlung am Hof, die wiederum zurückmünden sollte in eine synodales Inkraftsetzung des erzielten Konsens. Mit dieser geplanten Sequenz der Schritte, ausgeführt von verschiedenen Akteuren auf der kirchlichen und staatlichen Bühne lenkt die Doppelsynode von Rimini/Seleukia nochmals den Blick auf den Beitrag der Geschäftsabläufe, Verfahrensweisen und Verhandlungsformen, die bei der Entscheidungsfindung - auch zum Zweck theologischer Klarstellung - zusammenwirken, und die sowohl in synodalen Kontexten, als auch in benachbarten, aber unterscheidbaren, Rahmungen und zugehörigen Handlungsformen ausagiert werden.

Die vielfältigen, in verschiedenen Zusammenhängen überlieferten Nachrichten von den Vorbereitungen und Abläufen der Doppelsynode fügen sich nicht in allen Einzelheiten zu einem klaren Bild. Soweit es aber für das Verfahrensinteresse von Bedeutung ist, lassen sich verschiedene relevante Beobachtungen festhalten. ${ }^{41}$ Dabei gilt unser Augenmerk speziell den Verhaltensweisen der verschiedenen Teilnehmer, die aus den Dokumenten aufscheinen oder die in den Berichten der Historiker festgehalten sind.

An zwei verschiedenen Momenten des Geschehens illustrieren die Kirchenhistoriker ihre Berichte mit Hinweisen über die Aktivitäten der Bischöfe. Die Bischöfe Valens und Ursacius, begleitet und unterstützt von Germinius, and Gaius, brachten das

\footnotetext{
${ }^{39}$ Ausführlich und grundlegend für den gesamten hier ins Auge gefaßten Abschnitt kaiserlicher und kirchlicher Einigungsbemühungen - für die hier vorgelegte Untersuchung skizzenhaft auf wesentliche Grundschritte konzentriert - , s. Brennecke, Homöer 5-46; für die Teilsynode von Rimini insbesondere 5-40; Löhr, Entstehung 103-129. Vgl. zur Geschichte der Synode ferner Hefele-Leclerq I/2, 929-955, Simonetti, La crisi 314-325, Meslin, Les ariens 285-291; Barnes, Athanasius and Constantius, 145-149; sowie die detailreiche Rekonstruktion der komplexen Geschichte der Vorbereitung der Synode bei Löhr, Entstehung 93-98. Eine kurze Orientierung zu den Ereignissen bietet auch Ch. Pietri (bearb. v. J. Ulrich), Von der partitio des christlichen Kaiserreichs bis zur Einheit unter Konstantius: Arianerstreit und erster „Cäsaropapismus“, in: Die Geschichte des Christentums, Bd. 2: Das Entstehen der einen Christenheit (250-430), Freiburg/Br. u.a. 1996 (französisch Paris 1995), 386-391.

${ }^{40}$ Hil., coll.antiar. A 8 (93f. Feder).

${ }^{41}$ Zur Geschichte der sog. ersten Session, s. Löhr, Entstehung 103-13, bes. 112f. Die nachfolgende Untersuchung konzentriert sich allein auf die mit der westlichen Synode von Rimini verbundenen Ereignisse, doch wären analoge Beschreibungen der Geschehnisse von Seleukia möglich; vgl. zur Orientierung Brennecke, Homöer 40-59.
} 
Datierte Bekenntnis vor die Versammlung. ${ }^{42}$ Ihr Auftreten wird von Sozomenus in

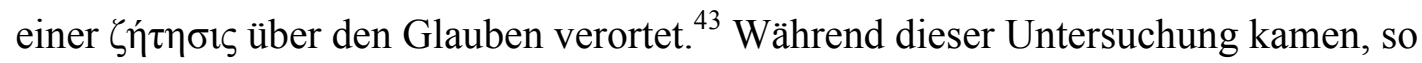
malt er aus, die Genannten in die Mitte, um den Text vorzustellen. Sie argumentierten auch unter Hinweis auf die Unterstützung des Kaisers für die (nicht nur politische) Notwendigkeit, ihn zu unterzeichnen und alle vorherigen Erklärungen beiseite zu setzen. Sie rieten von detaillierter Untersuchung der strittigen Terminologien ab

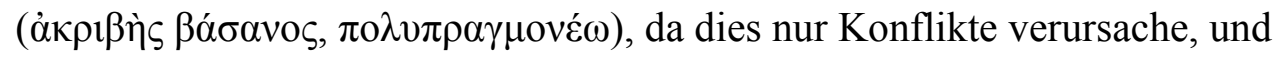
empfahlen das Bekenntnis als eine absichtsvoll schlichte Formel, damit „terminologische Neuerungen“ nicht Anlaß zur Einführung dialektischer Subtilitäten gäben. ${ }^{44}$ In der parallelen Passage berichtet Sokrates von der Verlesung des bis dahin absichtsvoll geheimgehaltenen Textes der fraglichen sog. vierten Sirmischen Formel. Man darf schließen, daß die Gruppe der Bischöfe einen für die Synode bis dahin unbekannten Text verlasen und ausführlich erläuterten, sowohl in theologischer wie in kirchenpolitischer Hinsicht. Nach Sokrates erhoben sich die Andersgesinnten im Anschluß an die Verlesung; dramatisierend hält er die Ablehnung der Gruppe in direkter Rede fest. ${ }^{45}$ Interessant ist hier der erzählerische Hinweis auf ihr Aufstehen. Will er andeuten, daß die Nizäner sich erhoben, um nach ihrer in dieser Form sicher fiktiven Ansprache hinauszugehen? Das Aufstehen suggeriert jedenfalls die Aufhebung der gemeinsamen Sitzung. Tatsächlich spaltete sich die Synode, doch sind Zeitpunkt und genaue Umstände nicht anderweitig überliefert. ${ }^{46}$ Von Sozomenos erfahren wir nichts über die Umstände der Zurückweisung des Textes; er konzentriert sich auf die verlangte und verweigerte Verurteilung der Arianischen Lehren als Motiv

\footnotetext{
${ }^{42}$ Socr., h.e. 2.37 .25 (155,10 Hansen); Soz., h.e. 4.17 .3 (163,8-13 Bidez/Hansen) - zu den Personen Hil., coll. Antiar. A 9.3 (96f. Feder) (=Doc. 58.5), Athan., syn. 11 (238,34-239,7 Opitz); vgl. Socr., h.e. 2.37.28 (155,19-20 Hansen); Soz., h.e. 4.17 .3 (163,8-9 Bidez/Hansen). Sokrates und Sozomenus nennen zusätzlich Auxentius von Mailand und Demophilus von Beröa als Mitglieder dieser Gruppe (ebd.). Allerdings sind beide nicht in den Verurteilungen durch die Synode erwähnt und darum wohl irrtümlich hier eingetragen; s. Doc. 58.5 mit den einführenden Bemerkungen.

${ }^{43}$ Soz., h.e. 4.17 .3 (163,7 Bidez/Hansen $)=$ Doc. 58.2. Konstantius hatte verlangt, daß die Fragen von Glaube und Kircheneinheit behandelt würden (Hil., coll. antiar. A 8.1.2. [94,4 Feder]: de fide atque unitate tractari); auch sonst ist die Beschäftigung mit den anstehenden Problemen in seinem Brief stets mit dem allgemeinen tractare beschrieben, das als solches keine spezifische Vorgehensweise determiniert (vgl. Hil., coll. antiar. 8.2.1 [94,16 Feder]).

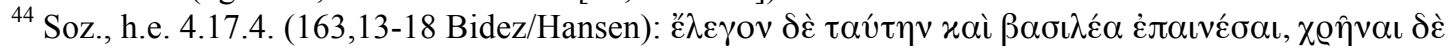

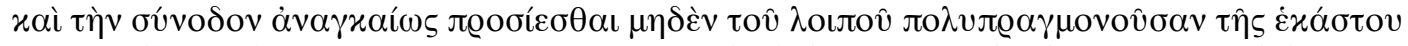

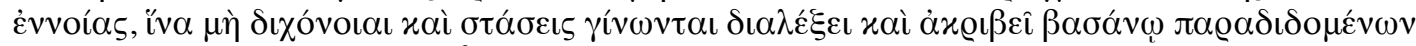

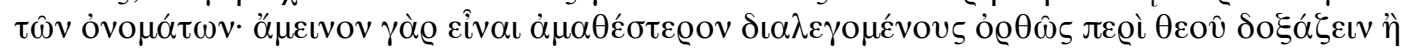

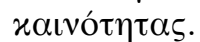

${ }^{45}$ Socr., h.e. 2.37.25-27 (155,10-18 Hansen).

${ }^{46}$ Sulp. Sev., chron. 2.41.5 (95,1-4 Halm) weiß von zwei separaten Tagungsstätten der ,Homöer' und der ,Nizäner'. Danach war es allerdings die Minderheit der Homöer, die auszog.
} 
für die Synode, die Gruppe der Bischöfe abzusetzen. ${ }^{47}$ Einen Protokollauszug in dem die Bischöfe der pronizänischen Teilsynode ihr Urteil über die Gruppe um Valens und Ursacius aussprachen bewahrt Hilarius auf. ${ }^{48}$ Es hat alle Anzeichen eines offiziellen Dokuments: Zunächst Datierung nach dem Konsuljahr und eine kurze einleitende Notiz über die Situation und den Anlaß der Synode; danach schlägt ein namentlich genannter Bischof die Verurteilung vor, und das Bischofskollektiv spricht sein placet aus; dies ist das eigentliche Urteil. Der aufgezeichnete Urteilsvorschlag des Bischofs Grecianus von Calle (von dem nichts weiter bekannt ist) besteht aus einer knappen narratio über die Beschäftigung der Synode mit Valens und Ursacius und mündet in die Aufforderung an die Bischöfe, ihr Urteil „abermals“ (iterum) auszusprechen und zu unterzeichnen, also eine in der Sache bereits gewonnenen Anschauung nun zu formalisieren. ${ }^{49}$ Der Hinweis auf ein wiederholtes Urteilen erinnert dabei einerseits wohl an Zurückweisungen der „Häretiker“ bei früheren Gelegenheiten ${ }^{50}$, dürfte aber speziell auf die Art und Weise anspielen, wie die Betroffenen auf der Synode selbst behandelt wurden. Denn, wir haben sie nicht zu unserer Gemeinschaft zugelassen (ad nostram communionem non admisimus) und sie mit unsere Stimme in ihrer Gegenwart verurteilt (voce nostra damnantes)“ “. ${ }^{51}$ In der partizipialen Verknüpfung formuliert der Bischof womöglich lediglich plerophor, ja tautologisch, wenn er NichtAufnahme in die Gemeinschaft und Exkommunikation je für sich nennt. Trotz der annähernden Synonymität beider Wendungen deutet sich gleichwohl eine mögliche Unklarheit bezüglich ihres Status bereits zu Eingang der Diskussionen an - speziell in der Frage nach ihrer Kirchengemeinschaft mit den übrigen Synodalen - und stellt sich darum die Frage nach einer Differenzierung unterschiedlicher Rollen im synodalen Geschehen. Wenn anfänglich keine förmliche Aufnahme der Gruppe in die Gemeinschaft erfolgte - womit anzunehmen ist, daß die Gemeinschaftsfrage eingangs unbehandelt und somit womöglich absichtsvoll in der Schwebe blieb -, so setzt im konkreten Fall ihr Auftreten in der Synode und ihre Vorlage und Diskussion des Textes nicht bestehende Gemeinschaft voraus, sondern wird ermöglicht in dem

\footnotetext{
${ }^{47}$ So. Athan., syn. 9,1 (236,16-19 Opitz); dazu kritisch Brennecke, Homöer 27-31.

${ }^{48}$ Hil., coll.antiar. A $9.3(96,16-97,13$ Feder $)=$ Doc. 58.5 .

${ }^{49}$ Hil., coll.antiar. A 9.3.2 (97,9-11 Feder): Nunc iterum quid uobis placet, iterum dicite ut singulorum suscriptione firmetur.

${ }^{50}$ Hil., coll.antiar. A 9.3.2 (97,7 Feder) = Doc. 58.5: Iam quidem heretici antehac nobis pronuntiati sunt...

${ }^{51}$ Hil., coll.antiar. A 9.3.2 (97,8f. Feder) = Doc. 58.5: Quos et ad nostram communionem non admisimus uoce nostra damnantes eos praesentes.
} 
Bemühen, die Möglichkeit zur Gemeinschaft zu sondieren. Wenn dies zutrifft, ist es von weitreichender Bedeutung für unser Verständnis synodalen Zusammenkommens und Kommunizierens und der Voraussetzungen und Vorbedingungen für synodales Handeln. Entweder hatte synodales Handeln nicht volle Gemeinschaft zur Voraussetzung, sondern nur die Bereitschaft, etwaige Grundlagen für Gemeinschaft zu untersuchen, und damit die Annahme, daß bestehende Differenzen im Prinzip überbrückbar waren. Oder aber die Beteiligung einzelner oder bestimmter Gruppen am synodalen Geschehen muß so verstanden werden, daß ihre Rolle in der Versammlung nicht unbedingt die eines gleichberechtigten Teilnehmers mit allen Rechten der Entscheidung und des Urteils sein muß. Wir müßten vielmehr mit einer differenzierteren und vielfältig abgestuften Bestimmung unterschiedlicher Rollen im synodalen Verfahrenskontext rechnen. ${ }^{52}$

Vielleicht weckt in dieser Blickrichtung die Formulierung des Sozomenos, wonach sie zur Vorlage des Textes ,in die Mitte“ kamen, zusätzliche Assoziationen. Man wird zwar seine Bemerkung ebenso wie die des Sokrates über das Aufstehen der Bischöfe nicht pressen und postulieren dürfen, daß sie auf historischer Information über die spezifischen Umstände des Geschehens beruhen. Ebensogut könnten sie als schriftstellerische Ausdruckmittel zur narrativen Verlebendigung verstanden werden, mit denen etwa Sozomenus die Bischöfe einfach „,ins Zentrum des Interesses“ rückt. Aber auch dann noch wäre in der Ausgestaltung und Verlebendigung der Szene etwas von den Erwartungen ihres Autors und seiner angenommenen Leser über synodale Vorgänge zu erkennen und müßte von derartigen Erwartungen ausgehend eine expressive Bedeutung des Agierens in einer Synode unterstellt werden.

So könnte Sozomenus' Formulierung schlicht ausdrücken, daß die Bischöfe vortraten, um den nächsten Punkt der Geschäftsordnung vorzubringen ${ }^{53}$, und insoweit synodale Konventionen und gängige Praktiken widerspiegeln. Doch ebenso wie der Hinweis des Sokrates auf das Aufstehen der Andersgesinnten über solche praktischen Usancen hinaus - üblicherweise erhob sich der Redende - mindestens darauf anzuspielen schien, daß sie sich von den anderen trennten und die Synode sich spaltete, so wäre auch vorstellbar, in Sozomenus' Plazierung der Bischöfe um Valens und Ursacius ,in

\footnotetext{
${ }^{52}$ Tatsächlich scheint sich dies bei anderer Gelegenheit zu bestätigen; s. u. zur Rolle des Valens in Rimini.

${ }^{53}$ Soz., h.e. 4.17 .2 (163,4-7 Bidez/Hansen). So versteht es der Übersetzer G.C. Hansen, Sozomenos: Historia Ecclesiastica/Kirchengeschichte, übersetzt und eingeleitet, 3 Bde. (Fontes Christiani 73/1-3), Trurnhout 2004, hier II. 493.
} 
der Mitte" eine Andeutung über ihre unklare Position und eine gewisse Ambivalenz ihrer Rolle abzulauschen. Über das Praktische hinaus hätte ihre Verortung ,in der Mitte“ womöglich weitere symbolische Resonanz. Beide Lesarten müssen einander nicht ausschließen. Im Gegenteil, die Positionierung von Einzelnen, die Sitzordnungen und Arrangements im Raum, das physische Interagieren und Gestikulieren, das die verbalen Interventionen begleitet, waren mindestens im selben Maße Ausdruck des Aushandelns und Darstellens von Status und Identität wie praktisch motiviert. ${ }^{54}$ Leider sind zeitgenössische Quellen, speziell Synodalprotokolle, eher schweigsam über diese Dimension synodalen Interagierens. Bei späteren Synoden, etwa in Chalcedon (451), wird aber gelegentlich sehr deutlich, daß Status und Rolle im Geschehen auch durch Plazierung ausgedrückt werden konnten, und speziell eine unsichere und angefochtene Mittelposition auch im räumlichen Arrangement erkennbar gemacht wurde. So finden sich während der ersten Sitzung dieses Konzils umstrittene Gestalten wie Dioskur von Alexandrien oder Theodoret von Kyrrhos in bestimmten Situationen ,in der Mitte““ ${ }^{55}$ Generell kommen Antragsteller immer wieder nach vorn und in die Mitte, um ihre Petitionen zu präsentieren. ${ }^{56}$ Tatsächlich harmonierte eine hypothetische Identifizierung der Rolle der genannten Bischöfe um Valens als Antragssteller nicht nur mit einer pragmatischen Beschreibung ihres Auftretens, sondern auch mit der Feststellung des Bischofs von Calle, wonach zu keinem (?) Zeitpunkt Gemeinschaft mit den Betreffenden aufgenommen worden war. Als Antragsteller könnten sie auch ohne

\footnotetext{
${ }^{54}$ Das Forschungsfeld der politischen und gesellschaftlichen Leistungsfähigkeit und der spezifischen Rationalität(en) von symbolischer Kommunikation - speziell, aber keineswegs nur in Mittelalter und früher Neuzeit - hat in den letzten Jahren einen enormen Aufschwung erfahren; es hier bibliographisch aufschließen zu wollen, verbietet sich. Im weiten Horizont vormoderner politischer Handlungs- und Gestaltungsmöglichkeiten bestimmt dieser Fragenkreis u.a. einen Arbeitsschwerpunkt des Sonderforschungsbereichs $496 »$ Symbolische Kommunikation und gesellschaftliche Wertesysteme vom Mittelalter bis zur französischen Revolution « und des Exzellenzcluster »Religion und Politik in den Kulturen der Vormoderne und Moderne« an der Universität Münster und zahlreiche damit verbundene Publikationen. Vgl. nur B. Stollberg-Rilinger, Symbolische Kommunikation in der Vormoderne. Begriffe - Thesen - Forschungsperspektiven, Zeitschrift für historische Forschung 31 (2004), 489-527. Für ein altkirchliches Beispiel des Versuchs, das Versammlungsformat einer Bischofsversammlung und die eigenen Rollenzuschreibung handelnd zu definieren, vgl. T. Graumann, Upstanding Donatists: Symbolic communication at the Conference of Carthage (411), Zeitschrift für antikes Christentum 15 (2011) 329-355.

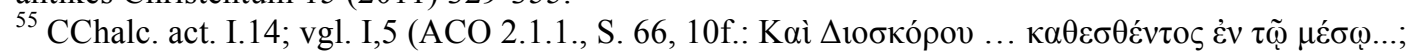

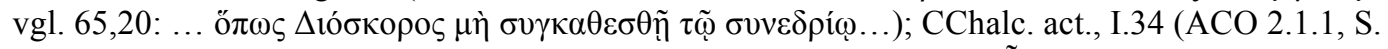

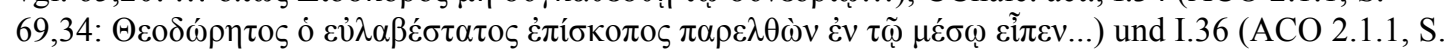

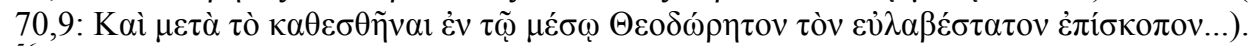

${ }^{56}$ Beispielsweise hält das Protokoll der Sitzung der Konstantinopler Synode von 448 die Anweisung fest, daß Ankläger und Angeklagter ,sich in die Mitte stellen sollen“ (CChalc., act. I, 475), oder auch, daß ein Notar zur Verlesung von Akten ,nach vorn und in die Mitte kam“ (CChalc., act. I.476). Ähnliche Platzanweisungen und Bewegungen ließen sich bei vielen Synoden dokumentieren.
} 
diese Vorbedingung agieren. ${ }^{57}$ Im fraglichen Kontext bei der Präsentation des Textes unterscheidet sich ihre Position jedenfalls von der aller anderen, die zur Beurteilung und Anerkenntnis oder Verwerfung ihres Vorschlages aufgerufen sind. Diese fungieren in der traditionellen synodalen Weise als situationsmächtige Richter, jene als Interessenvertreter, deren Aufgabe darin besteht, um Akzeptanz zu werben. Daß bei einem solchen Versuch auch die Frage nach persönlichem Vertrauen eine gewichtige Rolle spielt, zeigt eine spätere Zusammenkunft, auf der der Bruch mit den Genannten geheilt wird. Hier ist entscheidend, daß diese Rollendefinition nicht Gegenstand ausdrücklicher Benennung und Beratung sein mußte, sondern die Handlungsmuster als solche die schwierige Statusfrage pragmatisch lösten und zugleich für eine spätere Einigung mit den übrigen nützlich in der Schwebe hielten. Dort steht dann auch spezifisch die Möglichkeit zur Herstellung und Darstellung von Kirchengemeinschaft im Vordergrund dramatisch-symbolischen Agierens und wird das hier nur andeutungsweise erkennbare Augenmerk auf, und die eher beiläufige Beschäftigung mit dieser Frage gespiegelt und dabei zugleich schärfer konturiert. ${ }^{58}$

Nach der Zurückweisung des Textes, der Verurteilung seiner Verfechter und der darüber vollzogenen Spaltung der Synode in rivalisierende Teilversammlungen werden zunächst in (etwas frei interpretierter) Umsetzung der kaiserlichen Verfahrensanweisungen Delegationen der rivalisierenden Gruppen nach Konstantinopel entsandt, aber im thrakischen Nike festgehalten. Auf anhaltenden Druck, aber aus Gründen, die letztendlich im Dunkeln bleiben, hob die Delegation der hier behandelten Teilsynode die Verurteilung des Valens und seiner Genossen schließlich auf, nahm Gemeinschaft mit ihnen auf und unterschrieb eine geringfügig

\footnotetext{
${ }^{57}$ Man wird nicht mit letzter Gewißheit ausschließen wollen, daß sich dem Bischof diese Einschätzung eines problematischen Status der Freunde des Valens gleich zu Beginn des Geschehens erst ex eventu, im Nachgang der Ereignisse, erschloß. Daß Valens sich als „Antragsteller“ verstehen mußte, oder doch wenigsten so den anderen Bischöfen vor Augen stand, muß nicht zwangsläufig der ursprünglichen Konstellation in der entsprechenden Phase des Geschehens exakt entsprechen. Der zum Zeitpunkt seiner Intervention festgestellte Dissens und die sich anbahnende Verurteilung könnten Grecianus zu einer Neuinterpretation von dessen Rolle veranlaßt haben und seine rückblickenden Bemerkungen entsprechend färben. Dem so entstandenen Bild - verstärkt im Laufe der Zeit durch den stereotypen Arianismusvorwurf etwa in den polemischen Darstellungen des Athanasius - folgen die narrativen Dramatisierungen der Kirchenhistoriker. Bei einem hypothetischen anderen Ausgang, der Akzeptanz des Textes durch die Synode, wäre ihr Auftritt in der Mitte womöglich gar als Indiz einer ausgesprochenen Führungsrolle lesbar geworden, in der sie als Sachwalter des Willens der Gesamtsynode und ihre maßgeblichen Sprecher vor Augen stünden. So sind auch die physischgestischen Ausdrucksmittel nie in sich eindeutig, sondern bedarf ihre mögliche Signifikanz der Interpretation aus dem jeweiligen Kontext heraus.

${ }^{58}$ S.u. 000.
} 
modifizierte Version des eben noch verworfenen Texts. ${ }^{59}$ Die zurückkehrende

Delegation wurde von der Synode erst nach langem Widerstand und nicht ohne staatlichen Druck überhaupt empfangen, schließlich hatte sie völlig konträr zu ihrem Mandat gehandelt. Schlußendlich aber wurde doch in einem obskuren Manöver, das schon Zeitgenossen als den Betrug von Rimini bezeichneten, Einvernehmen erzielt. ${ }^{60}$ Die vielfach diskutierte komplizierte Frage, worin dieser Betrug bestand, soll hier nicht erneut behandelt werden. Das Interesse dieser Studie gilt allein den synodalen Formen und den Rollenangeboten und individuellen Handlungsmustern, mit deren Hilfe die erzielte Einigung ausagiert und dargestellt wurde. Dabei tritt das expressive Moment synodalen Entscheidens und Definierens von Rechtgläubigkeit besonders deutlich hervor. Die zu analysierende Szene belegt exemplarisch nicht allein die Bedeutung prozessualer Faktoren zur Herstellung von Einigung, sondern auch die Wichtigkeit seiner Darstellung, sowohl für die handelnd Beteiligten selbst, als auch vor dem Forum einer breiteren Öffentlichkeit.

Über die fraglichen, konventionell als zweite Sitzung von Rimini apostrophierten Vorgänge berichtet Hieronymus in der sog. Altercatio mit den Luciferanern. ${ }^{61}$ Dafür daß diese Einigung möglich wurde, trug nach seinem Bericht entscheidend bei, daß Valens sich bereit fand, arianische „Blasphemien“ zu verdammen. Anscheinend gab es Gerüchte, daß die Formel irgendeinen Betrug enthalte, und Valens verwarf die arianischen Sätze, um sich von solchen Verdächtigungen zu reinigen. ${ }^{62}$ Die Möglichkeit, einen theologischen Text zu akzeptieren, verlangt also nicht allen intellektuelle Valenz, sonder auch persönliches Vertrauen in seinen Verfechter (und

\footnotetext{
${ }^{59}$ Den Beschluß von Nike überliefert Hilarius, coll.antiar. A 5.3 (85f. Feder = Doc. 58.9), die Formel bei Thdt., h.e. 2.21.3-7 (145,4-146,12 Parmentier = Doc. 58.10); zu den Umständen vgl. Brennecke, Homöer 33f.

${ }^{60}$ Yves-Marie Duval, La « manœuvre frauduleuse » de Rimini: à la recherché du Liber adversus Vrsacium et Valentem, in: Hilaire et son temps. Actes du Colloque de Poitiers 29 Septembre - 3 Octobre 1968, Paris 1969, 51-103. (repr. in: idem., L'extirpation de l'Arianisme en Italie du nord et en occident: Rimini (359/60) et Aquilee (381), Hilaire de Poitiers (+367/8) et Ambroise de Milan (+397), Variorum Collected Studies Series CS 611, Brookfield, 1999); vgl. ferner Brennecke, Homöer 38f., Löhr, Entstehung 113-116. Für eine mögliche Unterscheidung mehrerer Zusammenkünfte unterschiedlicher Gruppierungen bei diesem Schlußakt der Synode von Rimini, s. Duval, manœuvre frauduleuse 61-63. Zur Frage der fraus jüngst nochmals I. Image, Nicene Fraud at the Council of Rimini, Studia Patristica 62 (2013) 313-322.

${ }^{61}$ Hier., c. Lucif. 17-18 (SC 437, 148,1-156,53 Canellis). Knappe Analyse, ohne Bezug auf die hier untersuchten Muster, bei Löhr, Entstehung 117-129.

${ }^{62}$ Hier., c.Lucif. 18 (150,5-152,10 Canellis): Denique, ipso in tempore, cum fraudem fuisse in expositione rumor populi uentilaret, Valens, Mursensis episcopus, qui eam conscripserat, praesente Tauro, praetorii praefecto, qui ex iussu regis synodo aderat, professus est se Arianum non esse et penitus ab eorum blasphemiis abhorrere.
} 
Mitautor). Allein da dies zunächst „,res secrete gesta“ geschah ${ }^{63}$, kam die

Gerüchteküche nicht zum Stillstand und wurde die Unruhe in der Öffentlichkeit nicht beseitigt.

Die vorgehaltene Heimlichkeit (secrete gesta) dieser ersten Erklärung des Valens ist erstaunlich und befremdlich. Sie kontrastiert mit der Beschreibung des seinerzeitigen ersten Vorschlags seiner Gruppe auf der sog. ersten Sitzung als öffentlich, also nicht in irgendwelchen Hinterzimmerverhandlungen, sondern in offener und formeller Konzilssitzung (publice in concilio). ${ }^{64}$ Demgegenüber erfolgte Valens' Versicherung nicht in einer Plenarsitzung der Synode, sondern hinter verschlossenen Türen. Der Bericht erwähnt die Anwesenheit des Prätorianerpräfekten Taurus. ${ }^{65}$ Es ließe sich konjizieren, daß Valens seine Erklärung vor dem Präfekten und in der Gegenwart nur einiger weniger anderer, etwa während einer Audienz, abgab. Der Präfekt dürfte durchaus konventionell für kaiserliche Beamte wichtige Amtsgeschäfte in einem separierten Raum abgehalten haben, der oftmals durch einen Vorhang vom Rest einer größeren Audienzhalle abgeteilt war. Womöglich erklärt ein solches Arrangement die Beschreibung der Erklärung des Valens als secrete gesta im Sinne einer durchaus üblichen Begrenzung des Zugangs der Öffentlichkeit zu wichtigen juristischadministrativen Amtsgeschäften besser als die Annahme konspiratorischer Heimlichkeit, deren Funktion und Nutzen fraglich bleiben müßte.

In jedem Fall bleiben die wahrgenommene Unzulänglichkeit dieses Verfahrens und das Bedürfnis festzuhalten, die Einigung über den engeren Kreis der kirchlichen und imperialen Amtsträger hinaus vor einer größeren Öffentlichkeit zu demonstrieren und zu inszenieren. Die Einigung in der Sache konnte nur auf kirchliche und gesellschaftliche Akzeptanz hoffen, konnte also nur plausibel Legitimität beanspruchen, wenn das Vertrauensverhältnis zwischen den Hauptakteuren sichtbar wiederhergestellt wurde und wenn insbesondere die Vertrauenswürdigkeit des Valens demonstrativ vorgeführt und bestätigt wurde. ${ }^{66}$ In ein Verhältnis sachlicher, d.h.

\footnotetext{
${ }^{63}$ Hier., c.Lucif. 18 (152,10 Canellis): Res secrete gesta opinionem uulgi non extinxerat.

${ }^{64}$ Diese Detail des Geschehens hält der Synodalbrief der Synode an Kaiser Konstantius II fest; Hil., coll.antiar. A 5.2.3 (82,6 Feder): ... quod offerebant publice in concilio ...

${ }^{65}$ Siehe PLRE 1, 879-880, s.v. Flavius Taurus.

${ }^{66}$ Für den Zusammenhang zwischen expressivem Handeln und der Herstellung und Erhaltung von Vertrauen sowie die zentrale Bedeutung des Vertrauensproblems bzw. der Beziehungsaspekte bei der Ermöglichung sozialen Handelns vgl. N. Luhmann, Vertrauen. Ein Mechanismus der Reduktion sozialer Komplexität, 4. Aufl. Stuttgart 2000 (Frankfurt/M. 1968). Vgl. für diese Dimension auch das Konzept des ,,sozialen Kapitals“ klassisch definiert in P. Bourdieu: Ökonomisches Kapital - Kulturelles Kapital - Soziales Kapital, in: R. Kreckel (Hg.): Soziale Ungleichheiten, Göttingen 1983, 183-198.
} 
theologischer, und politischer Übereinstimmung mit der Synode konnte er nur eintreten durch die Herstellung und Darstellung seiner sozialen Integration in den Kreis der legitimen kirchlichen Entscheidungsträger und der durch sie repräsentierten umfassenden kirchlichen (Heils-) Gemeinschaft und damit einhergehend durch die Umdeutung seiner persönlichen Rolle und seines Status im Gegenüber zu beiden. Die Legitimität des theologischen Konsens ereignet sich so im Vollzug seiner öffentlichen Darstellung und des handelnd, im Modus liturgisch gelebter und zelebrierter Kirchengemeinschaft, ausgedrückten Beweises der charakterlich-moralischen Festigkeit und Verläßlichkeit der wichtigsten Akteure und damit ihrer Befähigung, überhaupt mit dem Anspruch von Legitimität und Autorität ein solches Übereinkommen einzugehen.

Darum fand an einem der folgenden Tage eine erstaunliche Vorstellung statt. Das Geschehen darf mit Bedacht als „Vorstellung“ apostrophiert werden, da es überdeutliche Zeichen von Inszenierung aufweist. Auch geht es bei der fraglichen Zusammenkunft nicht darum, eine Entscheidung zu erzielen - dies war in der Sache bei dem Treffen vor dem Prätorianerpräfekten geschehen -, sondern sie öffentlich auszuagieren mit dem Ziel, das Bild von Einheit zwischen den Bischöfen und ihres völligen Übereinkommens über die Fragen des Glaubens zu kreieren und vorzuführen.

Eine große Menge, Bischöfe und Laien, versammelten sich in der Kirche. ${ }^{67}$ Damit sind bereits zwei bedeutsame Elemente angezeigt, die das nachfolgende Geschehen von den vorherigen Verhandlungen absetzen und in einen veränderten Rahmen einzeichnen: Zum einen wechselt die Situierung in den Raum der Kirche und zum anderen wird ausdrücklich die Präsenz einer weiteren Öffentlichkeit festgehalten. Schon damit ist klar, daß keine interne Exploration der Möglichkeit von Konsens oder der Prüfung theologischer Formeln ansteht, sondern gezielt das (herzustellende) einvernehmliche Verhältnis zwischen den Bischöfen zum Gegenstand öffentlicher Anschauung werden soll.

Ein Bischof namens Muzonius aus der Provinz Byzacena, ansonsten keiner der führenden Protagonisten der theologischen und kirchenpolitischen

\footnotetext{
${ }^{67}$ Hier., c.Lucif. 18 (152,11-12 Canellis): Itaque, alia die, in ecclesiam quae est apud Ariminum et episcoporum simul et laicorum turbis concurrentibus...
} 
Auseinandersetzungen, ${ }^{68}$ eröffnet das Prozedere; man hatte ihn dazu aufgrund seine Alters eingeladen. ${ }^{69}$ Dies zeigt bereits an, daß in dem nun eingeleiteten Verfahren konventionelle Statusmerkmale, nicht theologisches Fachwissen unterscheidend wirksam werden. Sein etwas unscharfer Verfahrensvorschlag ist, „die Dinge, die in der Öffentlichkeit kursierten und unsere Synode erreicht haben“" vorzulesen, damit was falsch sei verdammt werden könne. ${ }^{70}$ Als die Synode diesen Verfahrensvorschlag aufgreift, beginnt ein gewisser Bischof Claudius ${ }^{71}$ aus einem Text vorzulesen, der angeblich von Valens verfaßt sei. ${ }^{72}$ Die Textverlesung dient in merklicher Inszenierung von vornherein nur als Anlaß für das nachfolgende, eigentlich angezielte Geschehen; die Reaktionen sind keinesfalls spontan. Valens unterbricht die Verlesung laut rufend; er bestreitet, erstens, seine Autorschaft und spricht, zweitens, das Anathema über einen bestimmte Vorstellung (,Wenn einer leugnet, daß Christus der Herr, der Sohn Gottes vor den Zeiten aus dem Vater geboren wurde.... ${ }^{\text {(73) }}$ ) aus, die auf eine im soeben verlesenen Text formulierte Ansicht rekurrieren dürfte. Daraufhin respondieren die Bischöfe im Chor mit dem bestätigenden Ruf ,anathema sit!““ 74 Dieselbe Abfolge, ein von Valens ausgerufenes Anathema und die kollektiv antwortenden Rufe der Bischöfe ,anathema sit“, wiederholt sich sechsmal ${ }^{75}$, bevor die Bischöfe gemeinsam mit den versammelten Massen Valens mit Applaus und

\footnotetext{
${ }^{68}$ S. A. Mandouze, Prosopographie chrétienne du Bas-Empire, Bd. I: Afrique (303-533), Paris 1982, 768 s.v.

${ }^{69}$ Hier., c.Lucif. 18 (152,13-14 Canellis) = Doc. 58.12: Mizonius, episcopus prouinciae Byzacenae, cui propter aetatem primatus ab omnibus deferebatur, ita locutus est... Zur Textgestalt, s. A. Canellis, CSEL 79 B, p. 82 note critique ad 18,639.

${ }^{70}$ Hier., c.Lucif. 18 (152,15-18 Canellis $)=$ Doc. 58.12: Ea quae sunt iactata per publicum, et ad nos usque perlata, aliquem e nobis Sanctitati Vestrae legere praecipimus ut, quia sunt mala et ab auribus et a corde uestro abhorrere debent, una omnium uoce damnentur.

${ }^{71}$ Bischof der Provinz Pecenum in Italien, auch er keiner der Parteiführer und bestimmenden Theologen der Zeit. Vgl. Ch. Piétri und L. Piétri, Prosopographie chrétienne du Bas Empire. II: Italie (313-604), 2 Bde. Paris 1999, I. 448 s.v. Claudius I.

${ }^{72}$ Hier., c.Lucif. 18 (152, 20-22 Canellis): Itaque, cum Claudius, episcopus prouinciae Piceni, ex praecepto omnium, blasphemias quae Valentis ferebantur legere coepisset... Diesen Text zu identifizieren ist mir nicht gelungen; das wenige, was von Valens erhalten ist oder ihm zugeschrieben wird, besteht aus Briefen und beinhaltet keinen theologischen Traktat oder ähnliches Material. Vgl. CPL 682-687; M. Simonetti, Art. Valens of Mursa and Ursacius of Singidinum, Encyclopedia of the Early Church, ed. A. di Berardino (transl. A. Walford), 2 Bde, Cambridge 1992, II.858; B. Windau, Art. Valens von Mursa, $\mathrm{LACL}^{2}, 620 \mathrm{f}$.

${ }^{73}$ Hier., c.Lucif. 18 (152,23-24 Canellis): Si quis negat Christum Dominum, Dei filium, ante saecula ex Patre genitum, anathema sit!

${ }^{74}$ Hier., c.Lucif. 18 (152, 24-25 Canellis): Ab uniuersis consonatum est: Anathema sit!

${ }^{75}$ Hier., c.Lucif. 18 (152,25-154,34). Zur Frage eines ,zweiten“ Betrugs von Rimini in den Formulierungen der Anathematismen, s. Duval, manouevre frauduleuse, 98-103; Löhr, Entstehung 130-135.
} 
Fußgetrampel (tripudium ${ }^{76}$ ) unterbrechen. ${ }^{77}$ Die Freude der Bischöfe und der in der Kirche versammelten Öffentlichkeit findet also verbalen ebenso wie physischen Ausdruck, womit die prinzipielle Bedeutung von Verhalten und physischer Interaktion für die Kommunikation in den Synoden nochmals unterstrichen wird. Hieronymus fand in den Akten darüber hinaus Lobeshymen auf Valens und Ausdrücke des Bedauern darüber, ihn jemals als Häretiker verdächtigt zu haben, die er nicht im einzelnen wiedergibt. ${ }^{78}$

Hieronymus' Bericht unterstreicht aber, daß seine Darstellung anhand der Dokumente in öffentlichen und kirchlichen Archiven verifiziert werden könne und auch noch in lebendiger Erinnerung sei. Diese Akten, so beansprucht er, seien die Basis seines Berichts. ${ }^{79}$ Der bemerkenswerte Charakter der Ereignisse motiviert also eine besonders emphatische Betonung der Verläßlichkeit der Quellen, bzw. umgekehrt betrachtet: die Bedeutung, welche die Erzählung der Verläßlichkeit ihrer Quellen zumißt, zeigt an, daß das Geschehen als un- und außergewöhnlich wahrgenommen wurde.

Die Freudenszenen werden von Bischof Claudius, der bereits für das anfängliche Verlesen zuständig war, wieder in eine Art geordneten Geschäftsgang zurückgeleitet. ${ }^{80}$ Die öffentliche Aufführung der gefundenen Übereinstimmung zwischen Valens und den Bischöfen hat ihr Ende noch nicht ganz erreicht. Nachdem sich die Versammlung ein wenig beruhigt hat, hebt Claudius hervor, daß Valens bei seinen Verurteilungen noch einige Punkte übersehen habe, die gleichfalls zu verwerfen seien. Nur noch ein Anathema wird ausgeführt, bevor Hieronymus summarisch auf weitere im gleichen Stil erfolgte Verwerfungen verweist. ${ }^{81}$ Zwar spricht Hieronymus dabei von der Verwerfung solcher Sätze durch Valens ${ }^{82}$, aber das

\footnotetext{
${ }^{76}$ Der von Hieronymus gewählte Ausdruck bezeichnet ursprünglich das rituelle Tanzen von Priestern, spezifisch das dem Kriegsgotte Mars geweihte, nähert sich also Vorstellungen des Kriegstanzes an; s. OLD, sp. 1976 s.v.- Hieronymus kreiert so eine äußerst evokative Darstellung der Szene.

${ }^{77}$ Hier., c.Lucif. 18 (154,33-36 Canellis): In hoc uero, cuncti episcopi et tota simul ecclesia plausu quodam et tripudio Valentis uocem exceperunt.

${ }^{78}$ Hier., c.Lucif. 18 (154,42f. Canellis).

${ }^{79}$ Hier., c.Lucif. 18 (154,37-41 Canellis): Quod si quis a nobis fictum putauit, scrinia publica scrutetur. Plenae sunt certe Ecclesiarum chartae; recens adhuc rei memoria est. Supersunt homines qui illi synodo interfuerunt et, quod ueritatem firmet, ipsi Ariani haec ita ut diximus gesta non denegent. (156,51-53 Canellis): Quae si quis plenius discere cupit, in Ariminensis synodi actis reperiet, unde et nos ista libauimus.

${ }^{80}$ Hier., c.Lucif. 18 (154,43f. Canellis) ... idem Claudius qui supra legere coeperat, ait...

${ }^{81}$ Hier., c.Lucif. 18 (156,46-49 Canellis): Si quis dixerit Filium Dei esse quidem ante omnia saecula, sed non ante omne omnino tempus, ut ei aliquid anteferat, anathema sit! Dixerunt cuncti: Anathema sit! ${ }^{82}$ Hier., c.Lucif. 18 (156,49-51 Canellis): Multaque alia quae suspiciosa uidebantur, ad pronuntiationem Claudii, Valens condemnauit.
} 
einzig ausgeschriebene Beispiel - und implizit auch sein zusammenfassender Bericht - deuten auf eine signifikante Veränderung im Ablaufmuster hin.

Bischof Claudius bezeichnet in seinem Verfahrensvorschlag Valens als „Herrn und Bruder", behandelt ihn also als vollgültiges Synodenmitglied und impliziert bestehende Kirchengemeinschaft; er beantragt ausdrücklich weitere problematische Sätze gemeinschaftlich (in commune) zu verdammen. ${ }^{83}$ Tatsächlich ist auf die (von Hieronymus dem Protokoll entnommene) Anführung des ersten zu verwerfenden Satzes in dieser Sequenz nur notiert, daß alle (dixerunt cuncti) ${ }^{84}$ das Anathema aussprachen. Der Protokollauszug erwähnt nicht mehr, daß Valens zuerst und separat gesprochen habe wie zuvor. Selbst bei Hieronymus, dessen zusammenfassende Bemerkung ausschließlich Valens als denjenigen anführt, der solche Sätze verdammte, wird die tatsächliche Veränderung deutlich. Denn auch Hieronymus entnimmt dem Protokoll, daß die einzelnen Sätze jeweils von Claudius vorgebracht wurden - und eben nicht in der zuvor beschriebenen Weise von Valens. Claudius formuliert sicher nicht spontan, sondern verliest höchstwahrscheinlich die Anathematismen aus einem vorbereiteten Dokument. Damit handelt er in der gleichen Funktion und Rolle, in der er eingangs einen kritikwürdigen Text verlesen hatte. Dabei unterstreicht die Vorbereitung einer Liste von Anathematismen im übrigen nochmals den inszenierten Charakter des Geschehens; es läge nahe, auch Valens' frühere Interventionen auf dieselbe vorbereitete Anathematismenliste zurückzuführen. Während Claudius wiederum in der Rolle des Vorlesers agiert, hat sich die Rollenzuschreibung des Valens also fundamental verändert. Nachdem die Synode kurz zuvor so demonstrativ ihre Freude über die Übereinstimmung zwischen Valens und den anderen Bischöfen ausagiert hatte, konnte er nun als vollständig integriert angesehen werden und mit allen anderen zusammen handeln. Claudius' Benutzung des Bruder-Titels veranschaulicht den gleichen Statuswechsel. Klar erkennbar wird darin die zuvor nur undeutliche und hypothetisch festzustellende Möglichkeit zur Transformation der auf der Synode eingenommenen Rolle und des Status des Teilnehmers, und damit die prinzipielle Durchlässigkeit solcher Funktions- und Statusmerkmale im synodalen Handeln. Valens, der zunächst noch als unter Erklärungsnot stehender Einzelner im Gegenüber zum Kollektiv der Bischöfe sprach

\footnotetext{
${ }^{83}$ Hier., c.Lucif. 18 (154,44-156,46 Canellis): Sunt aliqua, quae subterfugerunt dominum et fratrem meum Valentem, quae, si uobis uidetur, ne qui scrupulus remaneat, in commune damnemus... ${ }^{84}$ S. Anm. 000 [84].
} 
und auftrat, dessen Rolle also anfangs unsicher und angefochten war, handelt nun ganz und gar als vollwertiges Mitglied der Synode und geht völlig in der Gruppe auf.

$$
* * *
$$

Hinter und in den spezifischen Umständen der beiden hier exemplarisch ausgewählten Bischofsversammlungen in der Mitte des vierten Jahrhunderts scheinen eine Vielzahl von auf den ersten Blick diversen Einzelbeobachtungen auf, die zusammengenommen die Bedeutung von Interaktions- und Verfahrensweisen und deren unausgesprochene kirchlich-sozialen Voraussetzungen für synodale Entscheidungsprozesse ins Bewußtsein heben.

Für die Frage nach der Möglichkeit verbindlicher „Orthodoxie“ zeigen sie die Verflechtung sachlich theologischer Argumentation - in Entwurf, Verlesung und Diskussion von Positionspapieren und Konsensdokumenten, in der streitigen Debatte um angemessene Schriftauslegung, in der Durchleuchtung von Begriffen und der verwerfenden Abgrenzung gegenüber abweichenden Sprach- und Argumentationsmustern - mit den Formen individuellen und kollektiven Rollenhandelns, dem agierenden Aushandeln von Statusfragen und den inszenatorisch-symbolischen Ausdrucksmöglichkeiten von Gemeinschaft, Konsens und Autorität. In ein spannungsreiches Verhältnis gesetzt sind diese zudem mit z.T. kaiserlich, (religions-)politisch vorgegebenen Verfahrensvorstellungen ebenso wie mit konventionellen Wahrnehmungen und Erwartungen der spätantiken Gesellschaft. So konnte näherhin inhaltlich theologische, speziell exegetische Diskussion in der unseren Erwartungen entsprechenden Form zwar in der Debatte zwischen Photin und Basilius beobachtet werden. Doch wurde hier klar, daß dieses Debattieren kein im engeren Sinne synodales Format darstellte, noch schlicht das Bemühen um intellektuelle Problemlösung repräsentierte, sondern als eine Mischung aus öffentlichem, quasi-akademischem Wettbewerb und verwaltungsgerichtlich orientierter Entscheidungsfindung außerhalb des synodalen Raums im engeren Sinne anzusiedeln war und die Mechanismen, Normen und Erwartungen jener Zusammenhänge spiegelte. Kaum je von den Beteiligten ausdrücklich reflektiert oder in den Dokumenten förmlich festgehalten, ist dennoch hier wie auch im zweiten Beispiel, der Synode von Rimini, das faktische Wirksamwerden von unausgesprochenen Rollenerwartungen an die Beteiligten und solchen an die 
Bedingungen der Legitimität des Geschehens erkennbar. Diese speisen sich aus verschiedenen institutionellen Kontexten, konventionellen zeitgenössischen intellektuellen Praktiken und gesellschaftlichen Entscheidungsprozessen. So entstehen hochkomplexe, z.T. ambivalente Verfahrensformen, in denen sich die Suche nach Orthodoxie jenseits, oder vielleicht besser: in Verlängerung und Transformation, des von Einzelnen und in polemischer Fachschriftstellerei durchbuchstabierten intellektuellen Ringens um konzeptionelle Klarheit und Überzeugung auch kollektiv und institutionell ausarbeitet.

Insbesondere die spezifischen Umstände der Synode von Rimini und die Wechselfälle zwischen ihrer sogenannten ersten und zweiten Sitzung erlauben in dieser Blickrichtung von einer planvollen, administrativ und institutionell ausgearbeiteten Choreographie von Verfahrensschritten zu sprechen. In dieser Rücksicht erwiesen Verfahrensformen sich nicht allein im jeweiligen Einzelgeschehen als signifikant, sondern war ein weit gespannter Zusammenhang von diversen Treffen mit verschiedenen Hauptakteuren, angefangen von der sirmischen Vorbereitungsgruppe bis hin zur öffentlichen Aufführung wiedergewonnener Eintracht in der sog. zweiten Sitzung von Rimini erkennbar, der als ein großer Spannungsbogen ansprechbar ist und als solche noch genauer verfahrensanalytisch untersucht werden müßte. Einem solchen primär institutionell nachzuzeichnenden Verfahrensbogen wohnt dabei ein zusätzliches Moment von kaum zu überschätzender Bedeutung inne. Gerade die zweite Sitzung von Rimini dokumentiert nämlich in seltener Klarheit und Expressivität die kritische Signifikanz symbolischen Handelns. Theologische Einigung oder auch Definition wurde hier an entscheidender Stelle nicht allein terminologisch-propositional diskutiert und fixiert, auch nicht allein kirchlichpolitisch ausgehandelt, sondern - das ist von erheblicher Wichtigkeit - vor allem öffentlich deklaratorisch zelebriert. Konsens und Einheit wurden performativ hergestellt in und durch ihre öffentliche symbolisch-rituelle Darstellung. Es zeigte sich so, daß das Verfahren als solches, die Inszenierung und öffentliche Zurschaustellung des Erreichten, ein entscheidendes Moment auch der Diskursivität und theologischen Arbeit von Synoden darstellt, ohne die ihre Leistungsfähigkeit für die Feststellung und Vermittlung der Legitimität bestimmter Ausdrücke von „Orthodoxie“" unverständlich bleibt. 